\title{
Modal Analysis of a Nonlinear Periodic Structure with Cyclic Symmetry
}

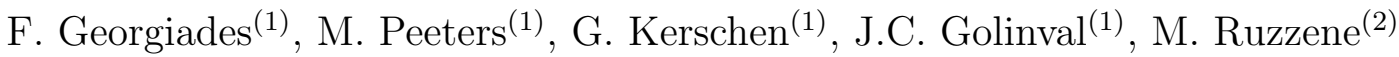

(1) Structural Dynamics Research Group Department of Aerospace and Mechanical Engineering

University of Liège, Liège, Belgium

E-mail: fotios.georgiades,m.peeters,g.kerschen,jc.golinval@ulg.ac.be

(2) School of Aerospace Engineering

Georgia Institute of Technology, Atlanta, USA

E-mail: massimo.ruzzene@ae.gatech.edu

Corresponding author:

G. Kerschen

Space Structures and Systems Lab (S3L)

Structural Dynamics Research Group

Department of Aerospace and Mechanical Engineering

University of Liège

1 Chemin des Chevreuils (B52/3), B-4000 Liège, Belgium.

Email: g.kerschen@ulg.ac.be

Phone: +32 4 3664852; Fax: +32 43664856 


\section{Abstract}

This paper carries out modal analysis of a nonlinear periodic structure with cyclic symmetry. The nonlinear normal mode (NNM) theory is briefly described, and a computational algorithm for the NNM computation is presented. The results obtained on a simplified model of a bladed assembly show that this system possesses a very complicated structure of NNMs, including similar and nonsimilar NNMs, nonlocalized and localized NNMs, bifurcating and internally resonant NNMs. Modal interactions that occur without necessarily having commensurate natural frequencies in the underlying linear system are also discussed.

\section{Introduction}

Modal analysis refers to the analysis of the dynamics of a vibrating structure in terms of its modal parameters. For linear systems, these parameters, termed the mode shapes, natural frequencies and damping ratios, can be computed from the governing equations of motion merely by solving an eigenvalue problem. A number of methods that can extract them directly from the system response have also been developed [1]. Modal analysis and testing of linear mechanical structures has been developed over the past 40-50 years, and the techniques available today are really quite sophisticated and advanced.

Modal analysis proved useful for analyzing the dynamics of linear periodic or nearly periodic structures (i.e., structures composed of a number of similar or identical elements coupled together, practical examples of which are bladed-disk assemblies and space antennas). In particular, energy localization phenomena occurring in slightly mistuned periodic structures were shown to exist due the presence of strongly localized modes $[2,3,4]$. Realizing that nonlinearity is a frequent occurrence in real-life applications, the dynamics of nonlinear periodic structures were also investigated in several studies. Wei and Pierre examined the effects of dry friction on a nearly cyclic structure using the harmonic balance method [5]. In a series of papers, Vakakis and co-workers demonstrated that, in contrast to the findings of linear theory, nonlinear mode localization may occur in perfectly cyclic nonlinear systems $[6,7,8,9]$. Other studies dealing with mode localization in nonlinear cyclic systems are $[10,11,12]$.

Because any attempt to apply traditional linear analysis to nonlinear systems is bound to failure, the nonlinear normal mode (NNM) theory is utilized herein for modal analysis of nonlinear periodic structures with cyclic symmetry. NNMs and asymptotic analysis were used in $[6,7,8,9]$ to examine the free and forced vibrations of nonlinear periodic systems with cyclic symmetry. In the present study, we revisit the free dynamics of such systems using the numerical algorithm for NNM computation proposed in $[13,14]$. This computational framework allows relaxing the assumptions of weak nonlinearity and/or weak coupling used in $[6,7,8,9]$. In addition, it is shown that modal interactions can take place in nonlinear systems without necessarily having commensurate natural frequencies in the underlying linear system. This dynamical phenomenon is rarely discussed in the 
literature.

The paper is organized as follows. In the next section, the two main definitions of an NNM are provided. The fundamental properties of NNMs are also described, and a numerical algorithm for their computation is introduced. In Section 3, the NNM theory is applied to a nonlinear cyclic system, and the resulting dynamics are analyzed in detail. The conclusions of the present study are summarized in Section 4.

\section{Nonlinear Normal Modes}

The free response of discrete mechanical systems is considered, assuming that continuous systems have been spatially discretized using, e.g., the finite element method. The equations of motion are

$$
\mathbf{M} \ddot{\mathbf{x}}(t)+\mathbf{K} \mathbf{x}(t)+\mathbf{f}_{n l}\{\mathbf{x}(t), \dot{\mathbf{x}}(t)\}=0
$$

where $\mathbf{M}$ is the mass matrix; $\mathbf{K}$ is the stiffness matrix; $\mathbf{x}, \dot{\mathbf{x}}$ and $\ddot{\mathbf{x}}$ are the displacement, velocity and acceleration vectors, respectively; $\mathbf{f}_{n l}$ is the nonlinear restoring force vector.

\subsection{Definitions}

NNMs were pioneered in the 1960s thanks to the seminal work of Rosenberg [15]. They were regarded as a theoretical curiosity until the beginning of the 1990s when they were given a new impetus through the efforts of Vakakis et al. [16, 17, 18] and Shaw and Pierre $[19,20]$.

There exist two main definitions of an NNM in the literature. During the normal mode motion of a linear conservative system, each system component moves with the same frequency and with a fixed ratio amongst the displacements of the components. Targeting a straightforward nonlinear extension of the linear normal mode (LNM) concept, Rosenberg defined an NNM as a vibration in unison of the system (i.e., a synchronous oscillation). This definition requires that all material points of the system reach their extreme values and pass through zero simultaneously and allows all displacements to be expressed in terms of a single reference displacement. One fundamental difference with linear systems is that the modal lines of nonlinear systems are generally curves, resulting from the nonlinear relationship between the coordinates during the periodic motion.

Shaw and Pierre proposed a generalization of Rosenberg's definition that provides a direct and elegant extension of the NNM concept to damped systems [20]. Based on geometric arguments and inspired by the center manifold technique, they defined an NNM as a twodimensional invariant manifold in phase space. Such a manifold is invariant under the flow (i.e., orbits that start out in the manifold remain in it for all time), which extends the invariance property of LNMs to nonlinear systems.

At first glance, Rosenberg's definition may appear restrictive in two cases: 
1. It cannot be easily extended to nonconservative systems.

2. In the presence of internal resonances (i.e., when two or more NNMs interact), some coordinates may have a dominant frequency component different than that of the other coordinates (e.g., some coordinates may vibrate faster than others). In this case, the system no longer vibrates in unison.

However, these two limitations can be circumvented. First, as shown in [13, 21], the damped dynamics can often be interpreted based on the topological structure and bifurcations of the NNMs of the underlying undamped system. Secondly, realizing that the motion is still periodic in the presence of internal resonances, Rosenberg's definition of an NNM can be extended to a (non-necessarily synchronous) periodic motion of the system. This extended definition is particularly attractive when targeting a numerical computation of the NNMs. As it will be shown in Section 2.3, the definition also enables the nonlinear modes to be effectively computed using advanced algorithms for the continuation of periodic solutions. This NNM definition is considered throughout the present study.

\section{$2.2 \quad$ Fundamental Properties}

A detailed description of NNMs and their fundamental properties is beyond the scope of this paper. However, for completeness, the frequency-energy dependence, as well as bifurcations and stability of NNMs are briefly reviewed in this section. For further details, the reader can refer to $[13,17,18]$.

A typical dynamical feature of nonlinear systems is the frequency-energy dependence of their oscillations. One important consequence is that the frequency response functions (FRFs) of nonlinear systems are no longer invariant. The modal curves and frequencies of oscillation of NNMs also depend on the total energy in the system. In contrast to linear theory, this energy dependence prevents the direct separation of space and time in the governing equations of motion, which complicates the analytical calculation of the NNMs.

Due to frequency-energy dependence, an appropriate graphical depiction of the NNMs is key to their exploitation. In this study, the representation of NNMs in a frequency-energy plot (FEP) is adopted. An NNM is represented by a point in the FEP, which is drawn at a frequency corresponding to the minimal period of the periodic motion and at an energy equal to the conserved total energy during the motion. A branch, represented by a solid line, corresponds to a family of NNM motions possessing the same qualitative features (e.g., the in-phase NNM motions of a 2DOF system). For illustration, a 2DOF system, the motion of which is governed by the equations

$$
\begin{aligned}
\ddot{x}_{1}+\left(2 x_{1}-x_{2}\right)+0.5 x_{1}^{3} & =0 \\
\ddot{x}_{2}+\left(2 x_{2}-x_{1}\right) & =0
\end{aligned}
$$

is considered, and its FEP is represented in Figure 1. The backbone of the plot is formed by two branches, which represent in-phase $(S 11+)$ and out-of-phase $(S 11-)$ synchronous 
NNMs. The FEP clearly shows that the nonlinear modal parameters have a strong dependence on the total energy in the system:

1. The frequency of both the in-phase and out-of-phase NNMs increases with the energy level, which reveals the hardening characteristic of the system.

2. The in-phase NNM tends to localize to the second DOF (i.e., it resembles a vertical curve), whereas the out-of-phase NNM localizes to the first DOF (i.e., it resembles an horizontal curve).

A second salient feature of nonlinear systems is that NNMs may interact during a general motion of the system. An energy exchange between the different modes involved may therefore be observed during the internal resonance. For instance, exciting a highfrequency mode may produce a large-amplitude response in a low-frequency mode. Internally resonant NNMs have no counterpart in linear systems and are generated through bifurcations. The FEP in Figure 1 does not seem to feature internally resonant NNMs. At higher energies, however, another branch of periodic solutions, termed a tongue, emanates from the backbone branch $S 11+$ (Figure 2). On this tongue, denoted $S 31$, there is a 3:1 internal resonance between the in-phase and out-of-phase NNMs. We note that other resonance scenarios (e.g., 2:1, 4:1, 5:1, etc.) exist in this seemingly simple system and are described in reference [13].

A third fundamental property of NNMs is that their number may exceed the number of DOFs of the system. Due to mode bifurcations, not all NNMs can be regarded as nonlinear continuation of normal modes of linear systems. Internally resonant NNMs are one possible example. Moreover, NNMs can be stable or unstable, which is in contrast to linear theory where all modes are neutrally stable. In this context, instability means that small perturbations of the initial conditions that generate the NNM motion lead to the elimination of the mode oscillation. Therefore, unstable NNMs are not physically realizable. In the present study, the stability analysis is performed numerically through the eigenvalues of the monodromy matrix. Bifurcations and stability are interrelated concepts, because a change in stability necessarily occurs through a bifurcation.

\subsection{Numerical Computation}

There have been very few attempts to compute NNMs using numerical methods (see, e.g., $[21,22,23,24])$. Interestingly, algorithms for the continuation of periodic solutions are really quite sophisticated and advanced $[25,26]$, yet they have not been fully exploited for the computation of nonlinear modes.

The numerical method considered for the NNM computation relies on two main techniques, namely a shooting technique and the pseudo-arclength continuation method. A detailed description of the algorithm is given in [14]. 


\subsubsection{Shooting Method}

The equations of motion of system (1) can be recast into state space form

$$
\dot{\mathbf{z}}=\mathbf{g}(\mathbf{z})
$$

where $\mathbf{z}=\left[\begin{array}{ll}\mathbf{x}^{*} & \dot{\mathbf{x}}^{*}\end{array}\right]^{*}$ is the $2 n$-dimensional state vector, with $(*)$ denoting the transpose operation, and

$$
\mathbf{g}(\mathbf{z})=\left(\begin{array}{c}
\dot{\mathbf{x}} \\
-\mathbf{M}^{-1}\left[\mathbf{K x}+\mathbf{f}_{\mathrm{nl}}(\mathbf{x}, \dot{\mathbf{x}})\right]
\end{array}\right)
$$

is the vector field. The solution of this dynamical system for initial conditions $\mathbf{z}(0)=\mathbf{z}_{0}=$ $\left[\begin{array}{ll}\mathbf{x}_{0}^{*} & \dot{\mathbf{x}}_{0}^{*}\end{array}\right]^{*}$ is written as $\mathbf{z}(t)=\mathbf{z}\left(t, \mathbf{z}_{0}\right)$ in order to exhibit the dependence on the initial conditions, $\mathbf{z}\left(0, \mathbf{z}_{0}\right)=\mathbf{z}_{0}$. A solution $\mathbf{z}_{p}\left(t, \mathbf{z}_{p 0}\right)$ is a periodic solution of the autonomous system (3) if $\mathbf{z}_{p}\left(t, \mathbf{z}_{p 0}\right)=\mathbf{z}_{p}\left(t+T, \mathbf{z}_{p 0}\right)$, where $T$ is the minimal period.

The NNM computation is carried out by finding the periodic solutions of the governing nonlinear equations of motion (3). In this context, the shooting method, probably one of the most popular numerical technique, solves numerically the two-point boundary-value problem defined by the periodicity condition

$$
\mathbf{H}\left(\mathbf{z}_{p 0}, T\right) \equiv \mathbf{z}_{p}\left(T, \mathbf{z}_{p 0}\right)-\mathbf{z}_{p 0}=\mathbf{0}
$$

where $\mathbf{H}\left(\mathbf{z}_{0}, T\right)=\mathbf{z}\left(T, \mathbf{z}_{0}\right)-\mathbf{z}_{0}$ is called the shooting function and represents the difference between the initial conditions and the system response at time $T$. Unlike forced motion, the period $T$ of the free response is not known a priori.

The shooting method consists in finding, in an iterative way, the initial conditions $\mathbf{z}_{p 0}$ and the period $T$ that realize a periodic motion. To this end, the method relies on direct numerical time integration and on the Newton-Raphson algorithm.

Starting from some assumed initial conditions $\mathbf{z}_{p 0}^{(0)}$, the motion $\mathbf{z}_{p}^{(0)}\left(t, \mathbf{z}_{p 0}^{(0)}\right)$ at the assumed period $T^{(0)}$ can be obtained by numerical time integration methods (e.g., Runge-Kutta or Newmark schemes). In general, the initial guess $\left(\mathbf{z}_{p 0}^{(0)}, T^{(0)}\right)$ does not satisfy the periodicity condition (5). A Newton-Raphson iteration scheme is therefore to be used to correct the initial guess and to converge to the actual solution. The corrections $\Delta \mathbf{z}_{p 0}^{(0)}$ and $\Delta T^{(0)}$ are found by expanding the nonlinear function

$$
\mathbf{H}\left(\mathbf{z}_{p 0}^{(0)}+\Delta \mathbf{z}_{p 0}^{(0)}, T^{(0)}+\Delta T^{(0)}\right)=0
$$

in Taylor series and neglecting higher-order terms (H.O.T.).

The phase of the periodic solutions is not fixed. If $\mathbf{z}(t)$ is a solution of the autonomous system (3), then $\mathbf{z}(t+\Delta t)$ is geometrically the same solution in phase space for any $\Delta t$. Hence, an additional condition, termed the phase condition, has to be specified in order to remove the arbitrariness of the initial conditions. This is discussed in detail in [14].

In summary, an isolated NNM is computed by solving the augmented two-point boundaryvalue problem defined by

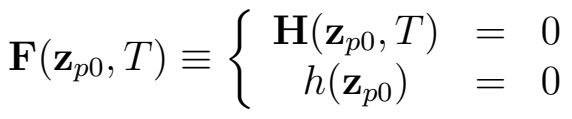


where $h\left(\mathbf{z}_{p 0}\right)=0$ is the phase condition.

\subsubsection{Continuation of Periodic Solutions}

Due to the frequency-energy dependence, the modal parameters of an NNM vary with the total energy. An NNM family, governed by equations (7), therefore traces a curve, termed an NNM branch, in the $(2 n+1)$-dimensional space of initial conditions and period $\left(\mathbf{z}_{p 0}, T\right)$. Starting from the corresponding LNM at low energy, the computation is carried out by finding successive points $\left(\mathbf{z}_{p 0}, T\right)$ of the NNM branch using methods for the numerical continuation of periodic motions (also called path-following methods) [26]. The space $\left(\mathbf{z}_{p 0}, T\right)$ is termed the continuation space.

Among the different methods for numerical continuation found in the literature, the socalled pseudo-arclength continuation method is used herein.

Starting from a known solution $\left(\mathbf{z}_{p 0,(j)}, T_{(j)}\right)$, the method consists in computing the next periodic solution $\left(\mathbf{z}_{p 0,(j+1)}, T_{(j+1)}\right)$ on the branch using a predictor step and a corrector step.

\section{Predictor step}

At step $j$, a prediction $\left(\tilde{\mathbf{z}}_{p 0,(j+1)}, \tilde{T}_{(j+1)}\right)$ of the next solution $\left(\mathbf{z}_{p 0,(j+1)}, T_{(j+1)}\right)$ is generated along the tangent vector to the branch at the current point $\mathbf{z}_{p 0,(j)}$

$$
\left[\begin{array}{c}
\tilde{\mathbf{z}}_{p 0,(j+1)} \\
\tilde{T}_{(j+1)}
\end{array}\right]=\left[\begin{array}{c}
\mathbf{z}_{p 0,(j)} \\
T_{(j)}
\end{array}\right]+s_{(j)}\left[\begin{array}{c}
\mathbf{p}_{z,(j)} \\
p_{T,(j)}
\end{array}\right]
$$

where $s_{(j)}$ is the predictor stepsize. The tangent vector $\mathbf{p}_{(j)}=\left[\mathbf{p}_{z,(j)}^{\mathrm{T}} p_{T,(j)}\right]^{\mathrm{T}}$ to the branch defined by (7) is the solution of the system

$$
\left[\begin{array}{c}
\left.\frac{\partial \mathbf{H}}{\partial \mathbf{z}_{p 0}}\right|_{\left(\mathbf{z}_{p 0,(j)}, T_{(j)}\right)} \\
\left.\frac{\partial h}{\partial \mathbf{z}_{p 0}}\right|_{\left(\mathbf{z}_{p 0,(j)}\right)}
\end{array}\right]\left[\begin{array}{l}
\mathbf{p}_{z,(j)} \\
p_{T,(j)}
\end{array}\right]=\left[\begin{array}{l}
\mathbf{0} \\
0
\end{array}\right]
$$

with the condition $\left\|\mathbf{p}_{(j)}\right\|=1$. This normalization can be taken into account by fixing one component of the tangent vector and solving the resulting overdetermined system using the Moore-Penrose matrix inverse; the tangent vector is then normalized to 1.

\section{Corrector step}

The prediction is corrected by a shooting procedure in order to solve (7) in which the variations of the initial conditions and the period are forced to be orthogonal to the predictor step. At iteration $k$, the corrections

$$
\begin{aligned}
\mathbf{z}_{p 0,(j+1)}^{(k+1)} & =\mathbf{z}_{p 0,(j+1)}^{(k)}+\Delta \mathbf{z}_{p 0,(j+1)}^{(k)} \\
T_{(j+1)}^{(k+1)} & =T_{(j+1)}^{(k)}+\Delta T_{(j+1)}^{(k)}
\end{aligned}
$$


are computed by solving the overdetermined linear system using the Moore-Penrose matrix inverse

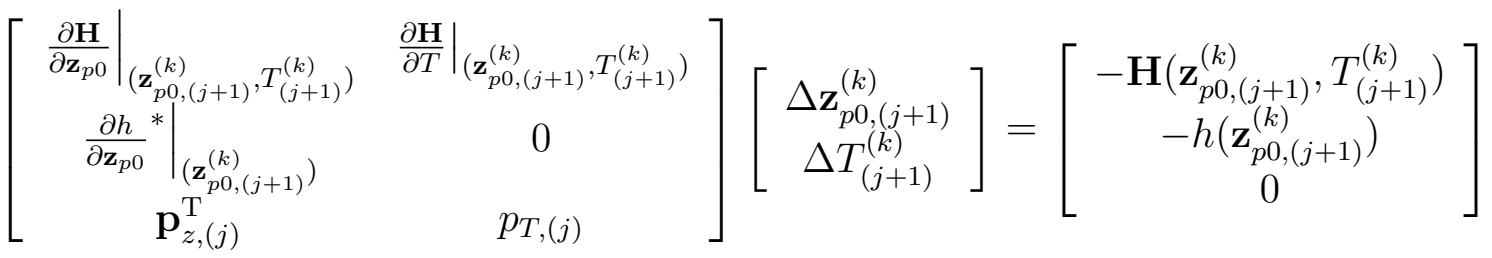

where the prediction is used as initial guess, i.e, $\mathbf{z}_{p 0,(j+1)}^{(0)}=\tilde{\mathbf{z}}_{p 0,(j+1)}$ and $T_{(j+1)}^{(0)}=\tilde{T}_{(j+1)}$. The last equation in (11) corresponds to the orthogonality condition for the corrector step.

This iterative process is carried out until convergence is achieved. The convergence test is based on the relative error of the periodicity condition:

$$
\frac{\left\|\mathbf{H}\left(\mathbf{z}_{p 0}, T\right)\right\|}{\left\|\mathbf{z}_{p 0}\right\|}=\frac{\left\|\mathbf{z}_{p}\left(T, \mathbf{z}_{p 0}\right)-\mathbf{z}_{p 0}\right\|}{\left\|\mathbf{z}_{p 0}\right\|}<\epsilon
$$

where $\epsilon$ is the prescribed relative precision.

\subsubsection{Algorithm for NNM computation}

The algorithm proposed for the computation of NNM motions is a combination of shooting and pseudo-arclength continuation methods, as shown in Figure 3. It has been implemented in the MATLAB environment. Other features of the algorithm such as the step control, the reduction of the computational burden and the method used for numerical integration of the equations of motion are discussed in [14].

So far, the NNMs have been considered as branches in the continuation space $\left(\mathbf{z}_{p 0}, T\right)$. As explained in Section 2.2, the NNMs are represented in a FEP in this study. This plot can be computed in a straightforward manner: (i) the conserved total energy is computed from the initial conditions realizing the NNM motion; and (ii) the frequency of the NNM motion is calculated directly from the period.

\section{Modal Analysis of a Nonlinear Cyclic Structure}

The objective of this section is to determine the essential features of the modal parameters of nonlinear periodic structures with cyclic symmetry. To this end, the modal shapes and frequencies of oscillation of the different NNM branches are computed from the free response of the undamped system.

The system considered throughout this paper is a simplified mathematical model of a bladed disk assembly. This model, though simplified, allows investigating interesting and complex dynamic phenomena related to the presence of nonlinearity. The lumped parameter model admits a single degree of freedom for each blade and includes a similarly 
simplified representation of the flexible disk. The bladed disk is composed of 30 sectors assembled with cyclic periodicity; a single sector is represented in Figure 4. Each sector is modeled using disk $(M)$ and blade $(m)$ lumped masses, coupled by linear $(k)$ and cubic $\left(k_{n l}\right)$ springs. The nonlinear springs can, for instance, be representative of geometrically nonlinear effects in slender blades. The disk masses are connected together by linear springs $K$. The equations of motion of this 60-DOF system are

$$
\begin{aligned}
m \ddot{x}_{i}+k\left(x_{i}-X_{i}\right)+k_{n l}\left(x_{i}-X_{i}\right)^{3} & =0 \\
M \ddot{X}_{i}+K\left(X_{i}-X_{i+1}\right)+K\left(X_{i}-X_{i-1}\right)+k\left(X_{i}-x_{i}\right)+k_{n l}\left(X_{i}-x_{i}\right)^{3} & =0
\end{aligned}
$$

for $i=1, \ldots, 30$, where $X_{31}=X_{1}, X_{0}=X_{30}$ (conditions of cyclic periodicity). $X_{i}$ and $x_{i}$ are the displacements of the disk and blade masses of the $i$ th sector, respectively. The values $M=1, m=0.3, K=1, k=1, k_{n} l=0.1$ are used in this study.

\subsection{Modal Analysis of the Underlying Linear System}

Before studying the nonlinear bladed disk assembly, the natural frequencies and mode shapes of the underlying linear system are first discussed. All bladed assemblies with circumferential symmetry exhibit single and double modes [27]:

- Double modes represent the majority. They have the same natural frequency and similar mode shapes. In fact, no unique mode shapes can be specified for these modes. Rather, it is sufficient to specify two suitably orthogonal shapes and to note that, when vibrating freely at that natural frequency, the structure can assume any form given by a linear combination of the two specified shapes. Further, at the corresponding natural frequency, the assembly can vibrate in any combination of $\cos n \theta$ and $\sin n \theta$ circumferential distributions of displacement around the assembly; i.e., in a shape of the form $\cos n \theta+\phi$. The mode shape is characterized by $\mathrm{n}$ nodal diameters since the displacement is constrained to be zero along n-equally spaced diametral lines. The mode shapes of a mode pair have mutually orthogonal nodal diameters.

- Single modes correspond to motion with all the blades undergoing, either in-phase (0 nodal diameter) or out-of-phase ( $\mathrm{N} / 2$ nodal diameters) motion.

The natural frequencies of the underlying linear bladed assembly are listed in Table 1 , where the modes are denoted by the integer pair $(n, m)$, which corresponds to the number of nodal circles $n$ and nodal diameters $m$ for the considered mode. In the model (13), the nodal circle parameter $n$ can only take the values $n=0$ or $n=1$, according to whether the blade and disk masses undergo in-phase or out-of-phase motion, respectively. One observes the existence of 28 pairs of double modes and 4 single modes. Figure 5 depicts four representative LNMs of the bladed assembly, namely mode $(0,15)$ and one mode of the mode pairs $(0,1),(1,5)$ and $(1,14)$. 


\subsection{Nonlinear Normal Modes (NNMs)}

Modal analysis of the nonlinear bladed assembly is carried out in this section using the previously described algorithm. NNM branches are computed by starting from the corresponding LNMs at low energy and gradually increasing the total energy in the system. These branches, termed backbone branches, are represented in Figure 6 and form the skeleton of the FEP. As we shall see, other NNM branches bifurcate from and coalesce into these backbone branches.

The first noticeable feature in Figure 6 is the frequency-energy dependence of the NNMs. The oscillation frequency of the modes with 1 nodal circle is strongly affected by the nonlinearities in the system. For these modes, the blade and disk masses vibrate in an out-of-phase fashion, which enhances nonlinear effects. On the other hand, the oscillation frequency of the modes with 0 nodal circle is much less affected. This is because the blade and disk masses vibrate in an in-phase fashion for these modes.

\subsubsection{Similar and Nonsimilar NNMs}

In addition to the dependence of their oscillation frequency, the NNMs may also have their modal shapes vary with the total energy in the system. According to Rosenberg's terminology [15], a similar NNM corresponds to an (energy-independent) straight modal line in the configuration space and occurs only in systems presenting certain spatial symmetries. A nonsimilar NNM corresponds to a curve in the configuration space, the shape of which varies with the total energy. Due to its symmetry properties, the system possesses both similar and nonsimilar NNMs. Two examples of similar NNMs in the bladed disk are the nonlinear extension of the LNMs with 0 nodal diameters, namely modes $(0,0)$ and $(1,0)$. Mode $(0,0)$ is a rigid-body mode, which is obviously unaffected by nonlinearity. The FEP of mode $(1,0)$ in Figure 7 clearly depicts that, while the NNM frequency is altered by the nonlinearities in the system, the modal shape remains unchanged.

Nonsimilar NNMs resemble the corresponding LNMs at low energy. The structure (i.e., the number of nodal circles and diameters) is preserved, and, as for the modes of the linear system, they mostly appear in pair. Nonsimilar NNMs in this system are either weakly, moderately or strongly affected by nonlinearity for increasing energy levels:

- Figure 8 represents a mode of the mode pair $(0,2)$, whose shape is almost energyindependent.

- Figure 9 shows that the NNMs of mode pair $(0,14)$ have a marked energy dependence.

- A remarkable property of the NNMs of mode $(1,14)$ is that the vibrational energy localizes to a limited number of sectors (4 in this case), the remaining of the system being virtually motionless (see Figure 10). The resulting spatial confinement of the energy causes the responses of some blades to become dangerously high and might lead to premature failure of the blades. For illustration, the time series 
corresponding to such an NNM motion are displayed in Figure 11. This localization phenomenon was also observed in linear mistuned bladed assemblies [28], but, here, it occurs even in the absence of structural disorder and direct interblade coupling. Localization is in fact the result of the frequency-energy dependence inherent to nonlinear oscillations, as discussed in reference [8].

All these NNM motions correspond to standing-wave motions in the sense that the system coordinates vibrate in a synchronous manner. They are represented by lines or curves in the configuration space. The phase condition used for their computation assumes that all initial velocities are zero. One therefore starts the motion from a maximum of the potential energy (see, e.g., Figure 11).

\subsubsection{Modal Interaction: Internally Resonant NNMs}

A first example of modal interaction is the 1:1 internal resonance between the two modes of a mode pair. This resonance scenario results in NNM motions which take the form of traveling waves and which are represented by ellipses in the configuration space. A detailed analytical study of these modes is given in reference [6]. Due to the existence of a phase difference between the coordinates, a different phase condition is considered for the NNM computation: only one initial velocity is set to zero, which is compatible with a traveling-wave motion. For instance, Figure 12 depicts the NNMs corresponding to 1:1 internal resonance between the modes of mode pair $(1,3)$. The mode structure is preserved in the sense that this traveling-wave motion also features one nodal circle and three nodal diameters. Representative time series are shown in Figure 13 and clearly highlight that the motion is no longer synchronous. In this particular case, the traveling wave is propagating in the anticlockwise direction, but its companion propagating in the clockwise direction also exists. These modes have an important practical significance, because they can be excited with an appropriate engine order excitation. This will be discussed in subsequent studies.

Other resonance scenarios can be observed in this system through the occurrence of tongues of internally resonant NNMs (see Section 2.2). Unlike backbone branches, tongues are localized to a specific region of the FEP. They bifurcate from the backbone branch of a specific mode and coalesce into the backbone branch of another mode, thereby realizing an internal resonance between the two modes. For instance, Figure 14 depicts a 3:1 internal resonance between modes $(0,6)$ and $(1,12)$ in the FEP. To better understand the resonance mechanism, the backbone of mode $(1,12)$ is represented at the third of its characteristic frequency (this is relevant, because a periodic solution of period $T$ is also periodic with period $3 T)$. This demonstrates that a smooth transition from mode $(0,6)$ to mode $(1,12)$ occurs on the tongue. A further illustration is that modes M1 and M2, which are the modes right after and before the coalescence of the two NNM branches, are almost identical.

During this 3:1 internal resonance, the system vibrates along a subharmonic NNM; i.e., an NNM motion characterized by more than one dominant frequency component. On the branch of mode $(0,6)$, the motion is characterized by one dominant frequency component, 
say $\omega$. As we move along the tongue from this branch, a third harmonic progressively appears, and the system vibrates with two dominant frequency components $\omega$ and $3 \omega$. As we progress further on the tongue, the third harmonic tends to dominate the component at the fundamental frequency, until this latter completely disappears. At this precise moment, a transition to mode $(1,12)$ is realized. This transition is illustrated in Figure 15 using time series representative of the NNM motion at three different locations on the tongue.

Surprisingly, the ratio of the linear natural frequencies of modes $(0,6)$ and $(1,12)$ is far from 3; it is equal to 2.47. A 3:1 internal resonance between the two modes can still be realized, because the frequency of mode $(0,6)$ increases much less rapidly than that of mode $(1,12)$, as shown in Figure 6 . This clearly highlights that NNMs can be internally resonant without necessarily having commensurate linear natural frequencies, a feature that is rarely discussed in the literature. Another interesting finding is that there is a countable infinity of branches of internally resonant NNMs in this system, similar to what was reported for a 2DOF system in [13].

\subsubsection{Additional Mode Bifurcations}

Another peculiar dynamical phenomenon that takes place in this system is mode splitting. For linear mistuned bladed assemblies and according to the degree of detuning, double modes with identical frequencies may also split into two different modes with distinct natural frequencies [27]. However, for nonlinear systems, due to mode bifurcations, a single NNM of a mode pair can split into two distinct NNMs (i.e., into NNMs with different oscillation frequencies and modal curves). A direct consequence is that a mode pair can bifurcate into two mode pairs, and the number of NNMs exceeds the number of DOFs of the system, as mentioned in Section 2.2. For illustration, the splitting of one mode of the mode pair $(1,5)$ is depicted in Figure 16. Clearly, after the bifurcation, two NNM branches exist and are characterized by different oscillation frequencies and modal curves.

Other examples of mode bifurcations are shown in Figures 17 and 18 for the mode pair $(1,14)$ and mode $(1,15)$, respectively. Mode pair $(1,14)$ undergoes a bifurcation during which a new NNM branch is generated. Interestingly, this branch is characterized by modes that are localized to two sectors only. Even more interesting is the bifurcation of mode $(1,15)$, which generates mode shapes that are localized to only one sector. Depending on their stability properties, these NNMs may be excited in practice, giving rise to potentially harmful motion that must be accounted for.

\subsubsection{Mode Stability}

In the present study, stability analysis is performed numerically through the eigenvalues of the monodromy matrix, which is a by-product of the proposed algorithm. NNM stability is an important concept, because it dictates whether or not a mode is physically realizable. For instance, Figure 19 shows the stability properties of mode $(1,15)$. From 
very low energies to energies slightly above $1 J$, the NNMs are stable, and, hence, physically realizable. From this latter energy, a stability change occurs, and the NNMs become unstable. This stability change occurs through a bifurcation, which coincides exactly with the generation of the branch of NNMs localized to one sector in Figure 18.

\section{Concluding Remarks}

In this study, the free vibrations of a nonlinear periodic structure with cyclic symmetry were examined using the NNM theory. The NNMs were computed numerically by combining a shooting technique with pseudo-arclength continuation. One advantage of this approach is that it provides the capability for analysis of strongly nonlinear regimes of motion.

A very complicated structure of NNMs, including similar and nonsimilar NNMs, nonlocalized and localized NNMs, bifurcating and internally resonant NNMs, was observed. One important finding of this study is that modal interactions can occur without necessarily having commensurate natural frequencies in the underlying linear system. Furthermore, a countable infinity of such modal interactions were shown to exist in this system.

Further research will investigate how forced resonances of nonlinear periodic structures relate to the topological structure of their NNMs. This will provide useful insight into the forced dynamics, which is of particular practical significance.

\section{References}

[1] D.J. Ewins, Modal Testing: Theory, Practice and Application, Research Studies Press LTD (2nd edition), Hertfordshire, 2000.

[2] C. Pierre, E.H. Dowell, Localization of vibrations by structural irregularity, Journal of Sound and Vibration 114 (1987), 549-564.

[3] O.O. Bendiksen, Localization phenomena in structural dynamics, Chaos, SOlitons and Fractals 11 (2000), 1621-1660.

[4] M.P. Castanier, C. Pierre, Using intentional mistuning in the design of turbomachinery rotors, AIAA Journal 40 (2002), 2077-2086.

[5] S.T. Wei, C. Pierre, Effects of dry friction damping on the occurrence of localized forced vibrations in nearly cyclic structures, Journal of Sound and Vibration 129 (1989), 397-416.

[6] A.F. Vakakis, Dynamics of a nonlinear periodic structure with cyclic symmetry, Acta Mechanica 95 (1992), 197-226. 
[7] A.F. Vakakis, C. Cetinkaya, Mode localization in a class of multidegree-of-freedom nonlinear-systems with cyclic symmetry, SIAM Journal on Applied Mathematics 53 (1993), 265-282.

[8] A.F. Vakakis, T. Nayfeh, M.E. King, A multiple-scales analysis of nonlinear, localized modes in a cyclic periodic system, Journal of Applied Mechanics 60 (1993), 388-397.

[9] M.E. King, A.F. Vakakis, A very complicated structure of resonances in a nonlinear system with cyclic symmetry: nonlinear forced localization, Nonlinear Dynamics 7 (1995), 85-104.

[10] M.E. King, P.A. Layne, Dynamics of nonlinear cyclic systems with structural irregularity, Nonlinear Dynamics 15 (1998), 225-244.

[11] C.N. Folley, Bifurcation and Symmetry in Cyclic Structures, Ph.D. Thesis, Purdue University, 1999.

[12] S. Samaranayake, G. Samaranayake, A.K. Bajaj, Resonant vibrations in harmonically excited weakly coupled mechanical systems with cyclic symmetry, Chaos, Solitons and Fractals 11 (2000), 1519-1534.

[13] G. Kerschen, M. Peeters, J.C. Golinval, A.F. Vakakis, Nonlinear normal modes, Part I: A useful framework for the structural dynamicist, Mechanical Systems and Signal Processing, in review.

[14] M. Peeters, R. Viguié, G. Sérandour, G. Kerschen, J.C. Golinval, Nonlinear normal modes, Part II: Practical computation using numerical continuation techniques, Mechanical Systems and Signal Processing, in review.

[15] R.M. Rosenberg, On nonlinear vibrations of systems with many degrees of freedom, Advances in Applied Mechanics 9 (1966), 155-242.

[16] A.F. Vakakis, Analysis and Identification of Linear and Nonlinear Normal Modes in Vibrating Systems, Ph.D. Dissertation, California Institute of Technology, 1990.

[17] A.F. Vakakis, L.I. Manevitch, Y.V. Mikhlin, V.N. Pilipchuk, A.A. Zevin, Normal Modes and Localization in Nonlinear Systems, John Wiley \& Sons, New York, 1996.

[18] A.F. Vakakis, Non-linear normal modes and their applications in vibration theory: an overview, Mechanical Systems and Signal Processing 11 (1997), 3-22.

[19] S.W. Shaw, C. Pierre, Non-linear normal modes and invariant manifolds, Journal of Sound and Vibration 150 (1991), 170-173.

[20] S.W. Shaw, C. Pierre, Normal modes for non-linear vibratory systems, Journal of Sound and Vibration 164 (1993), 85-124.

[21] Y.S. Lee, G. Kerschen, A.F. Vakakis, P.N. Panagopoulos, L.A. Bergman, D.M. McFarland, Complicated dynamics of a linear oscillator with a light, essentially nonlinear attachment, Physica D 204 (2005), 41-69. 
[22] J.C. Slater, A numerical method for determining nonlinear normal modes, Nonlinear Dynamics 10 (1996), 19-30.

[23] E. Pesheck, Reduced-order modeling of nonlinear structural systems using nonlinear normal modes and invariant manifolds, PhD Thesis, University of Michigan, Ann Arbor, 2000.

[24] R. Arquier, S. Bellizzi, R. Bouc, B. Cochelin, Two methods for the computation of nonlinear modes of vibrating systems at large amplitudes, Computers \& Structures 84 (2006), 1565-1576.

[25] E. Doedel, AUTO, Software for Continuation and Bifurcation Problems in Ordinary Differential Equations, 2007.

[26] R. Seydel, Practical Bifurcation and Stability Analysis, from Equilibirum to Chaos, Springer-Verlag, 2nd Edition, 1994.

[27] D.J. Ewins, Structural Dynamics Characteristics of Bladed Assemblies, Lecture Series 1992-06 on Vibration and Rotor Dynamics, Von Karman Institute for Fluid Dynamics, 1992.

[28] M.P. Castanier, C. Pierre, Modeling and analysis of mistuned bladed disk vibration: status and emerging directions, Journal of Propulsion and Power 22 (2006), 384-396. 


\begin{tabular}{|c|c|c|c||c|c|c|c|}
\hline Mode & $\begin{array}{c}\text { Nodal } \\
\text { circles }\end{array}$ & $\begin{array}{c}\text { Nodal } \\
\text { diameters }\end{array}$ & $\begin{array}{c}\text { Freq. } \\
\text { (rad/s) }\end{array}$ & Mode & $\begin{array}{c}\text { Nodal } \\
\text { circles }\end{array}$ & $\begin{array}{c}\text { Nodal } \\
\text { diameters }\end{array}$ & $\begin{array}{c}\text { Freq. } \\
\text { (rad/s })\end{array}$ \\
\hline 1 & 0 & 0 & 0.000 & 31 & 1 & 0 & 2.082 \\
2,3 & 0 & 1 & 0.183 & 32,33 & 1 & 1 & 2.084 \\
4,5 & 0 & 2 & 0.363 & 34,35 & 1 & 2 & 2.092 \\
6,7 & 0 & 3 & 0.536 & 36,37 & 1 & 3 & 2.104 \\
8,9 & 0 & 4 & 0.700 & 38,39 & 1 & 4 & 2.123 \\
10,11 & 0 & 5 & 0.850 & 40,41 & 1 & 5 & 2.147 \\
12,13 & 0 & 6 & 0.985 & 42,43 & 1 & 6 & 2.178 \\
14,15 & 0 & 7 & 1.103 & 44,45 & 1 & 7 & 2.215 \\
16,17 & 0 & 8 & 1.202 & 46,47 & 1 & 8 & 2.258 \\
18,19 & 0 & 9 & 1.282 & 48,49 & 1 & 9 & 2.304 \\
20,21 & 0 & 10 & 1.346 & 50,51 & 1 & 10 & 2.350 \\
22,23 & 0 & 11 & 1.394 & 52,53 & 1 & 11 & 2.394 \\
24,25 & 0 & 12 & 1.428 & 54,55 & 1 & 12 & 2.431 \\
26,27 & 0 & 13 & 1.452 & 56,57 & 1 & 13 & 2.460 \\
28,29 & 0 & 14 & 1.465 & 58,59 & 1 & 14 & 2.478 \\
30 & 0 & 15 & 1.470 & 60 & 1 & 15 & 2.485 \\
\hline
\end{tabular}

Table 1: Natural frequencies of the underlying linear bladed assembly. 


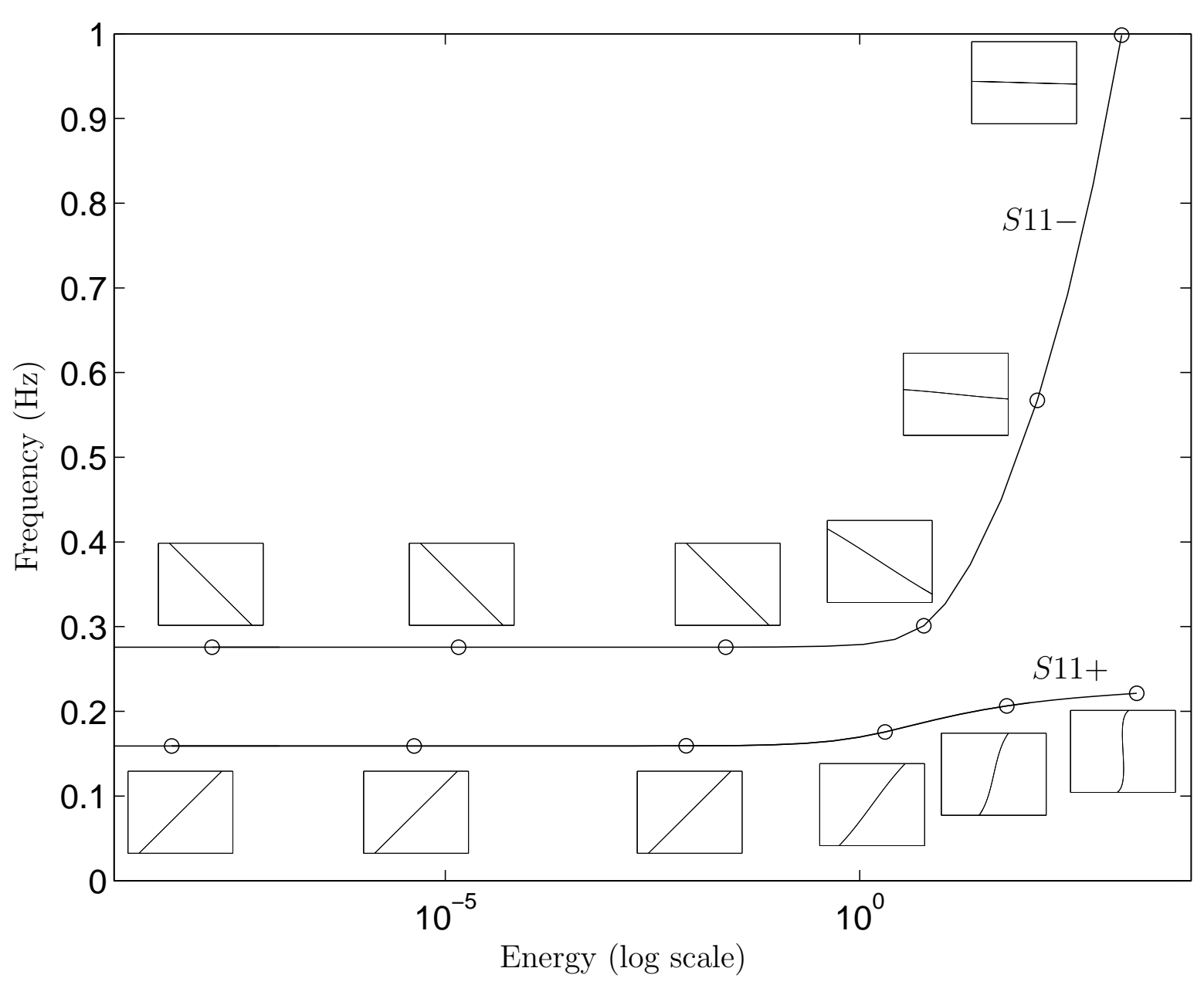

Figure 1: Frequency-energy plot of system (2). NNM motions depicted in the configuration space are inset. The horizontal and vertical axes in these plots are the displacements of the first and second DOFs, respectively; the aspect ratio is set so that increments on the horizontal and vertical axes are equal in size. 


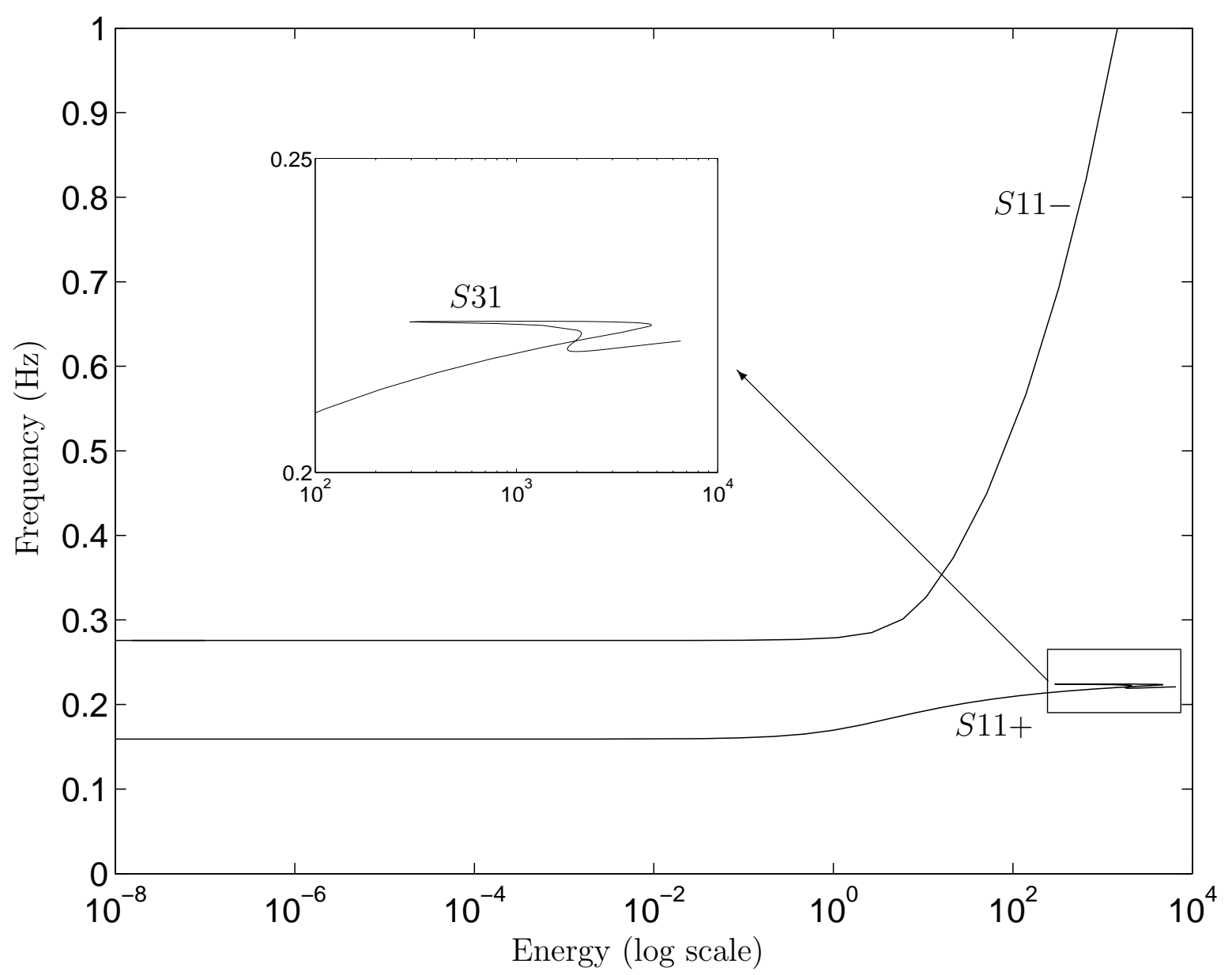

Figure 2: Frequency-energy plot of system (2) featuring a 3:1 internal resonance between the in-phase and out-of-phase NNMs. 


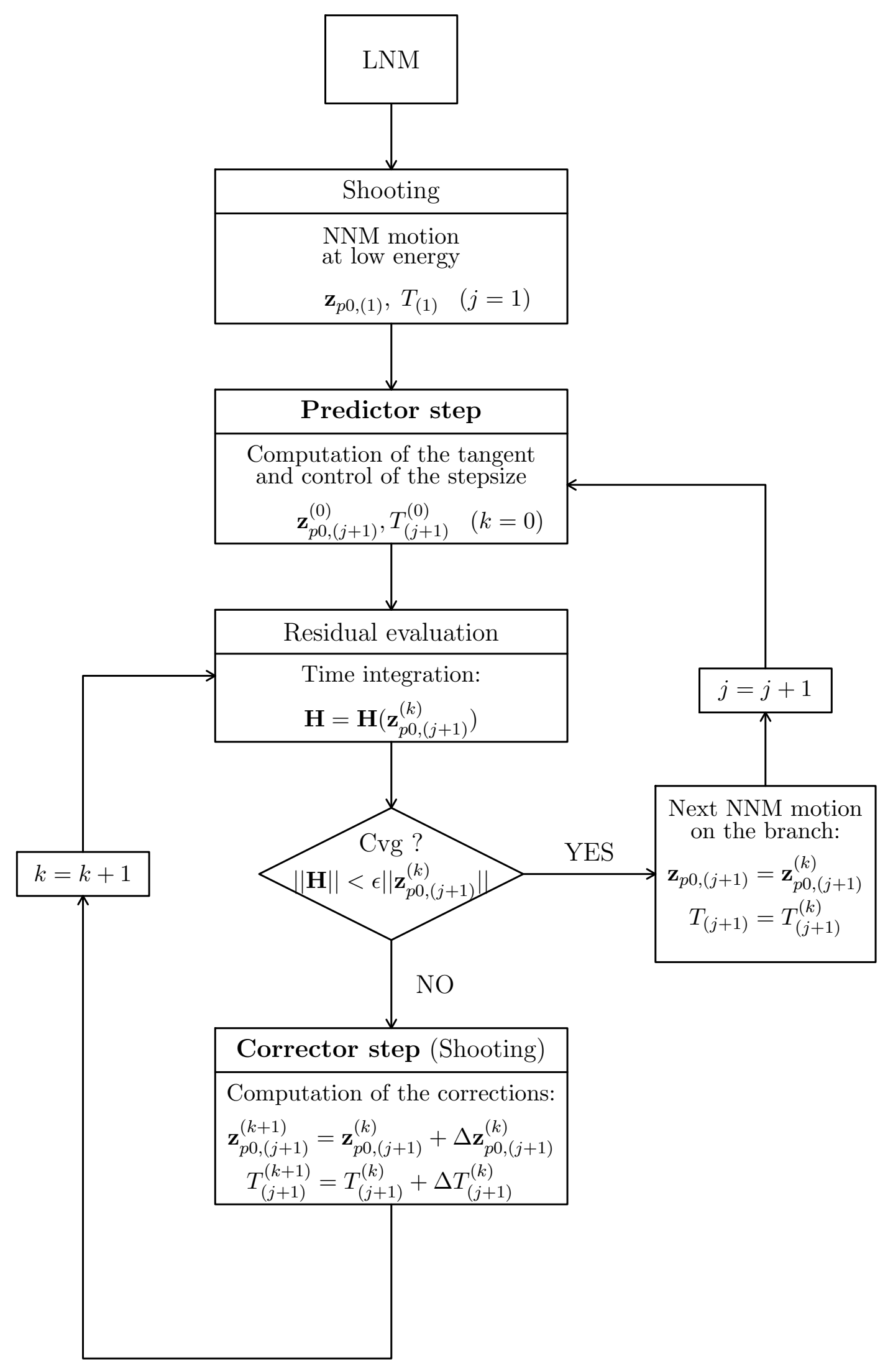

Figure 3: Algorithm for NNM computation. 


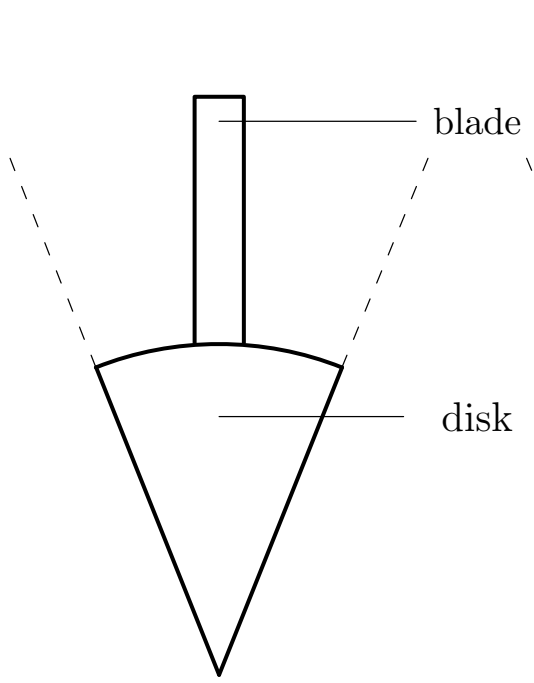

(a)

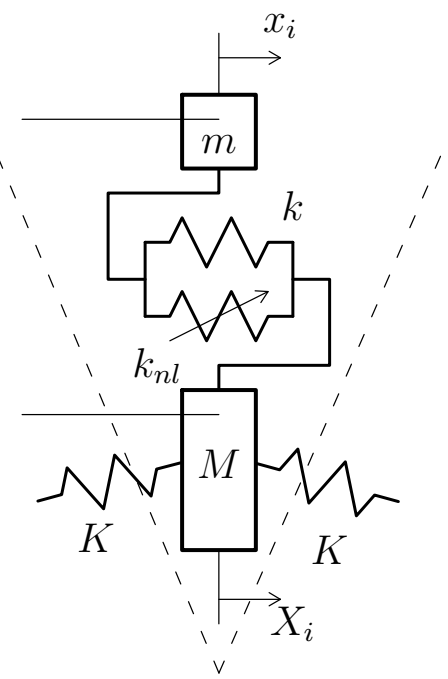

(b)

Figure 4: One sector of the nonlinear bladed disk assembly. (a) continuous structure; (b) discrete model. 

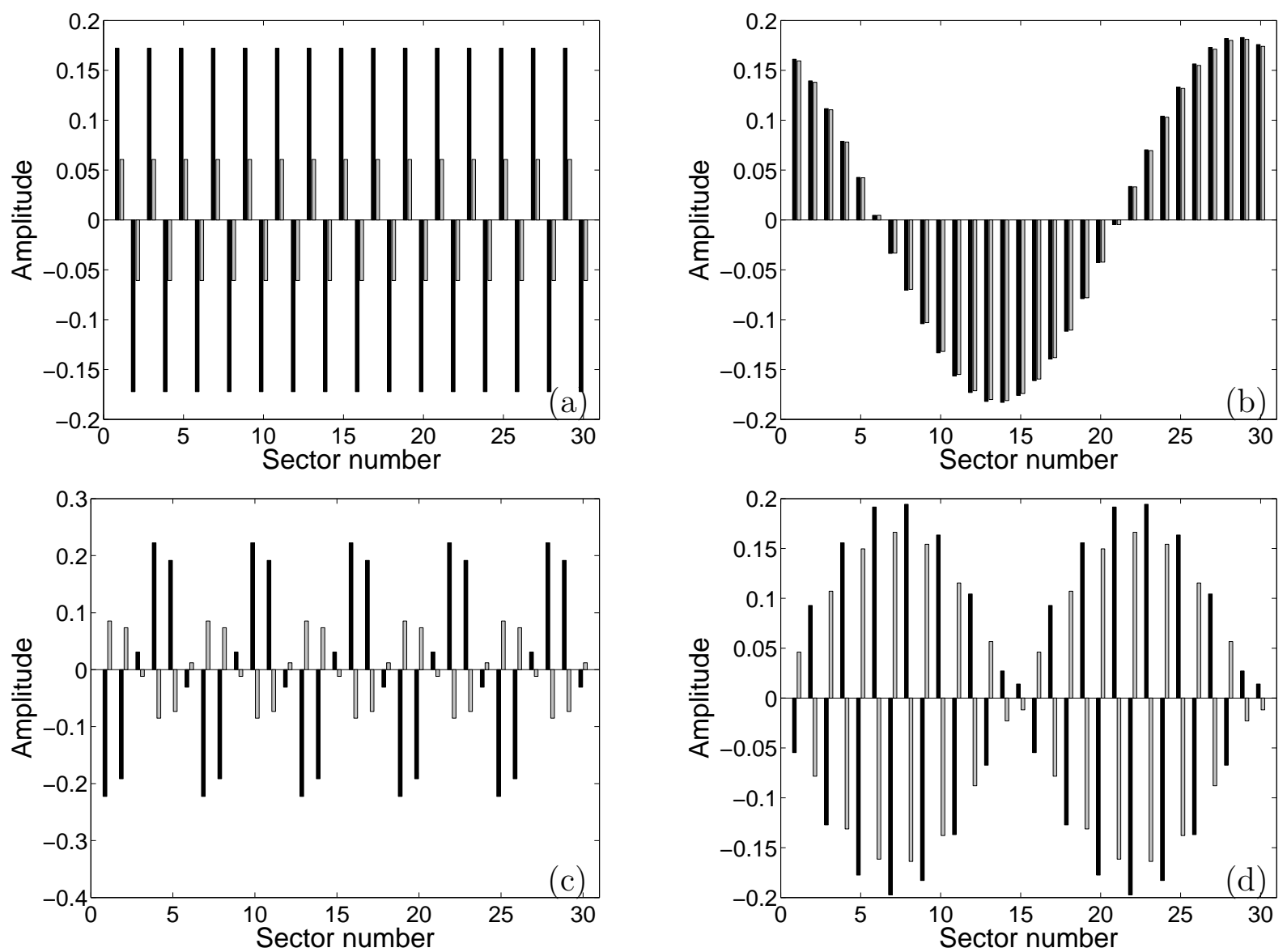

Figure 5: Representative LNMs of the bladed assembly; the blade and disk masses are shown in black and grey, respectively. (a) Mode $(0,15)$ and one mode of the mode pair (b) $(0,1) ;(\mathrm{c})(1,5)$ and $(\mathrm{d})(1,14)$. 


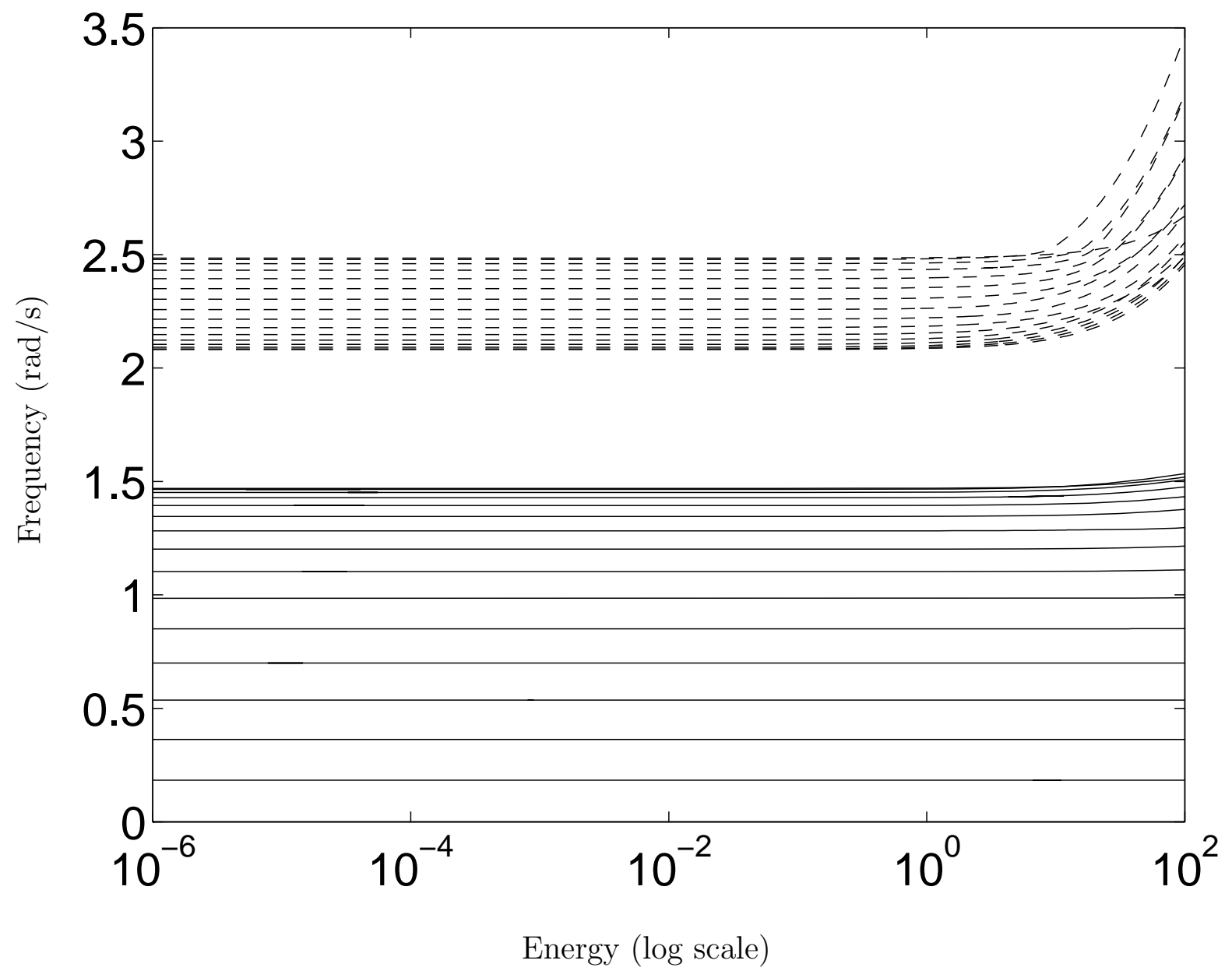

Figure 6: Evolution of the NNM frequencies with the total energy in the system. Solid lines: NNM with 0 nodal circle; dashed lines: NNM with 1 nodal circle. 


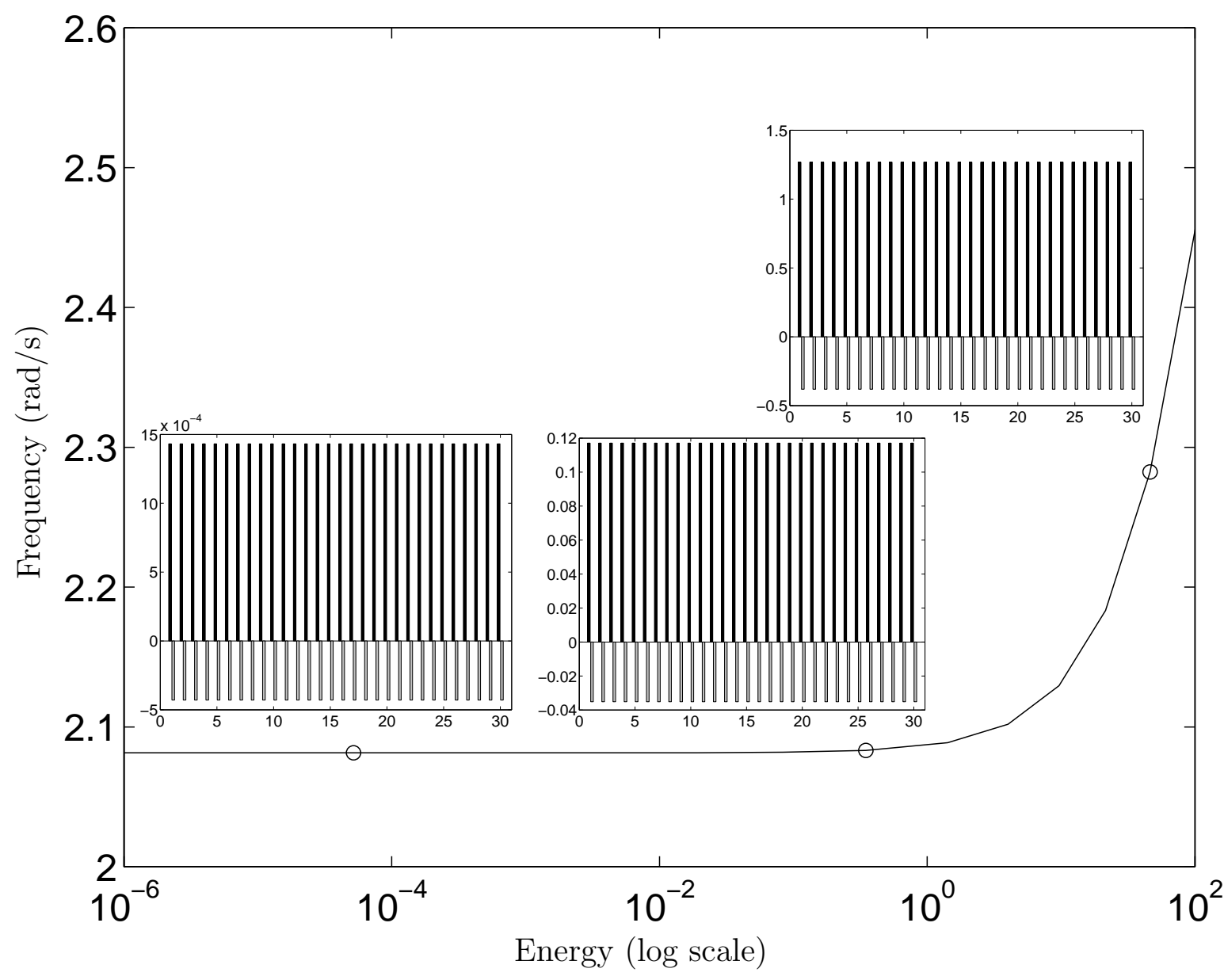

Figure 7: FEP of mode (1,0). NNMs represented by bar graphs are inset; they are given in terms of the initial displacements that realize the periodic motion (with zero initial velocities assumed). The blade and disk masses are shown in black and grey, respectively. 


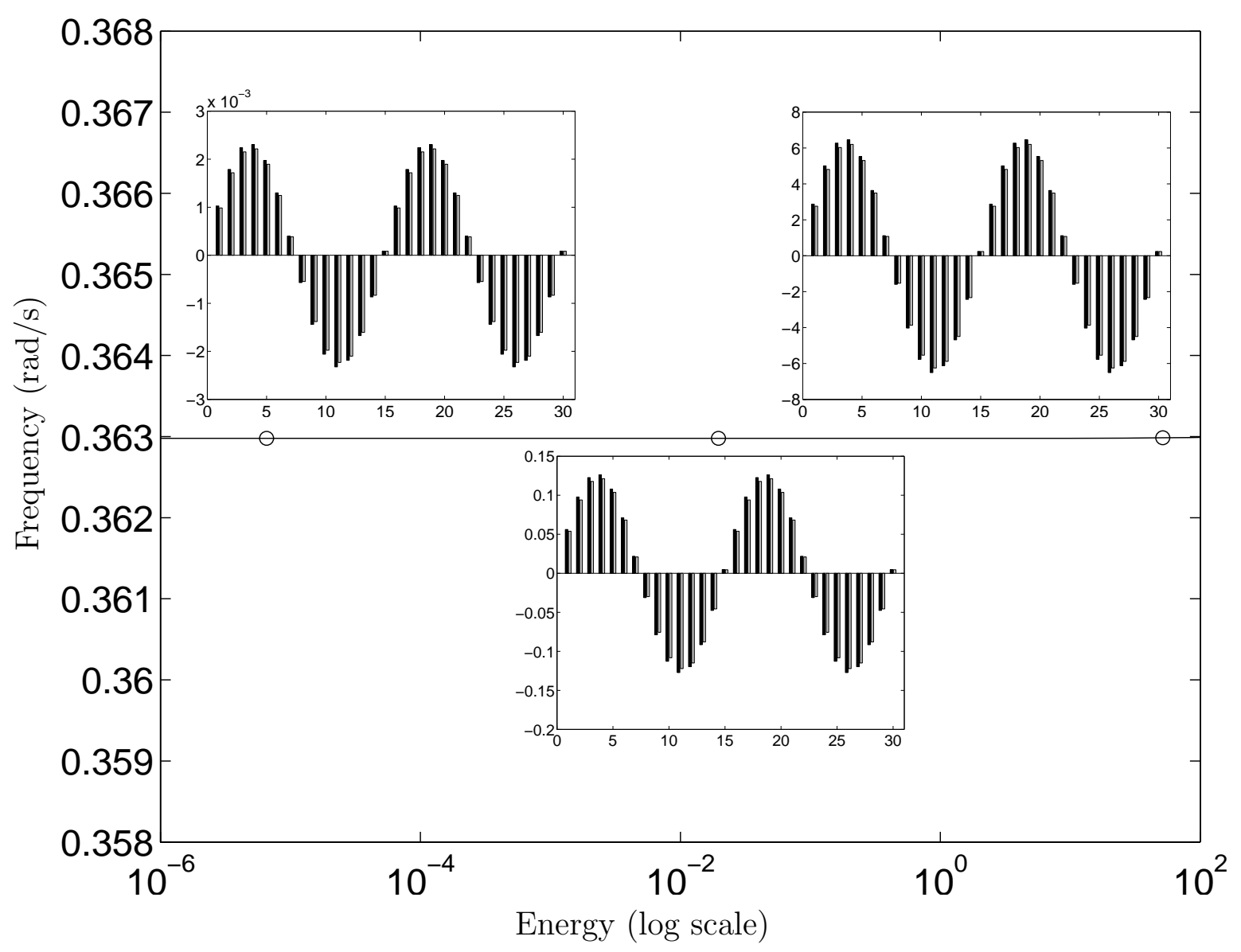

Figure 8: FEP of one mode of the mode pair $(0,2)$. NNMs represented by bar graphs are inset; they are given in terms of the initial displacements that realize the periodic motion (with zero initial velocities assumed). The blade and disk masses are shown in black and grey, respectively. 


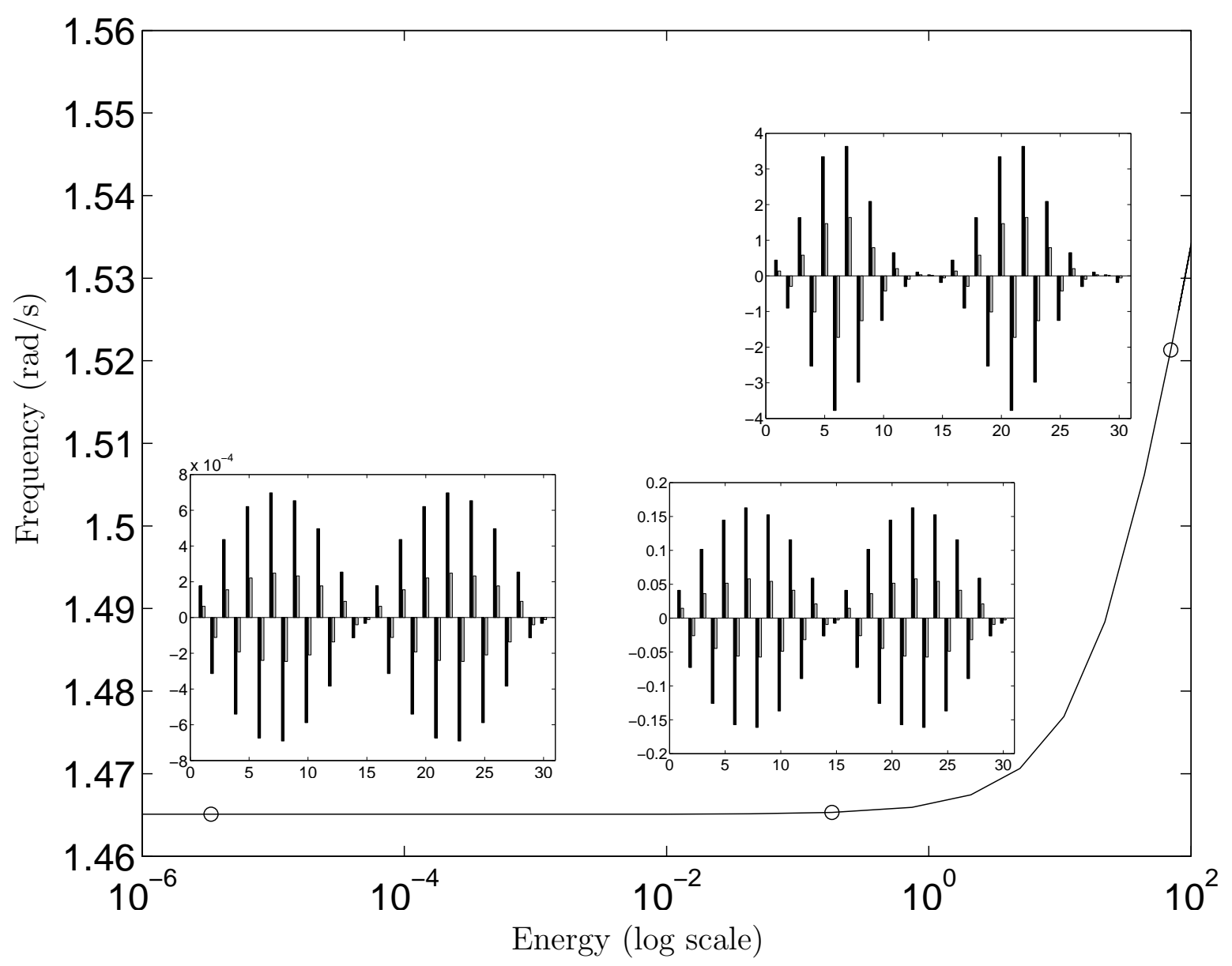

Figure 9: FEP of one mode of the mode pair $(0,14)$. NNMs represented by bar graphs are inset; they are given in terms of the initial displacements that realize the periodic motion (with zero initial velocities assumed). The blade and disk masses are shown in black and grey, respectively. 


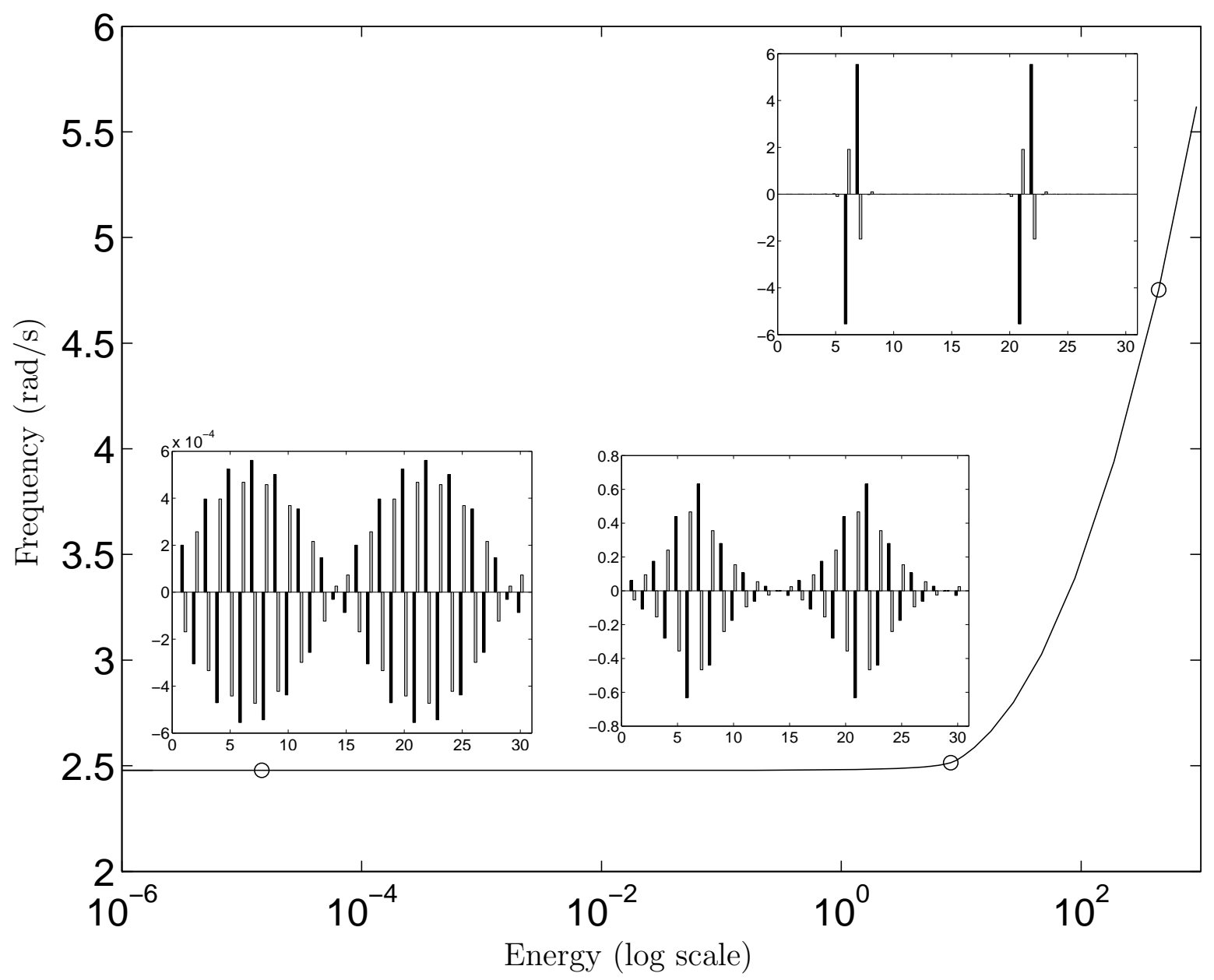

Figure 10: FEP of one mode of the mode pair $(1,14)$. NNMs represented by bar graphs are inset; they are given in terms of the initial displacements that realize the periodic motion (with zero initial velocities assumed). The blade and disk masses are shown in black and grey, respectively. 


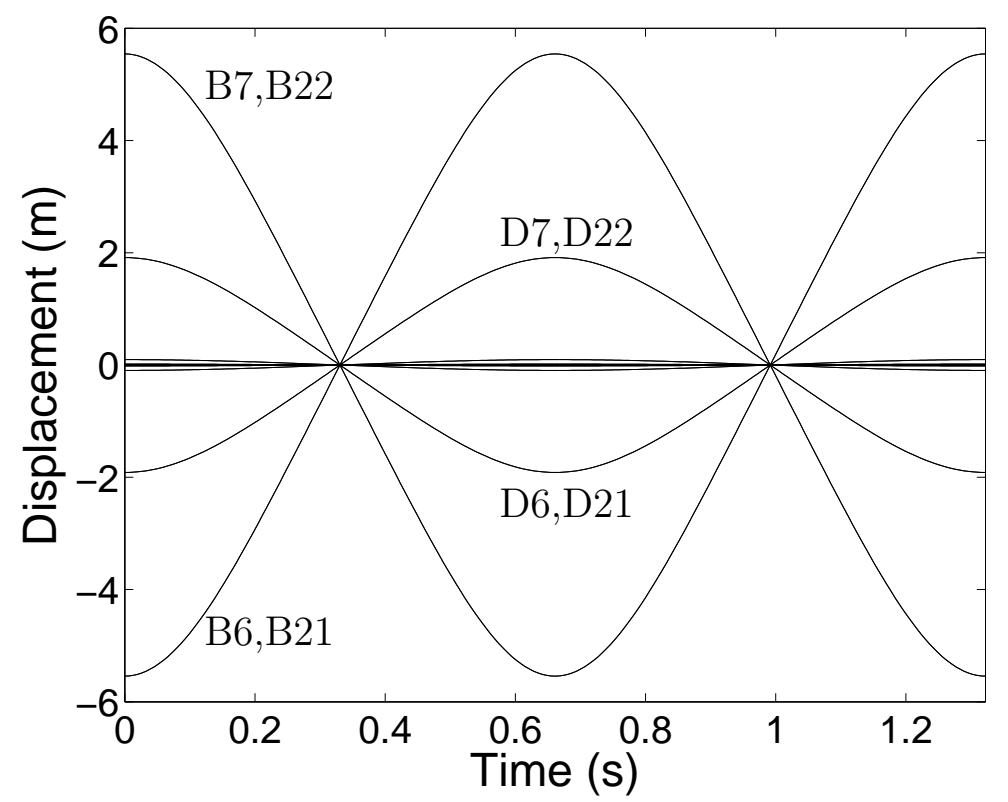

Figure 11: Time series corresponding to the localized NNM motion of mode $(1,14)$ (see Figure 10). 


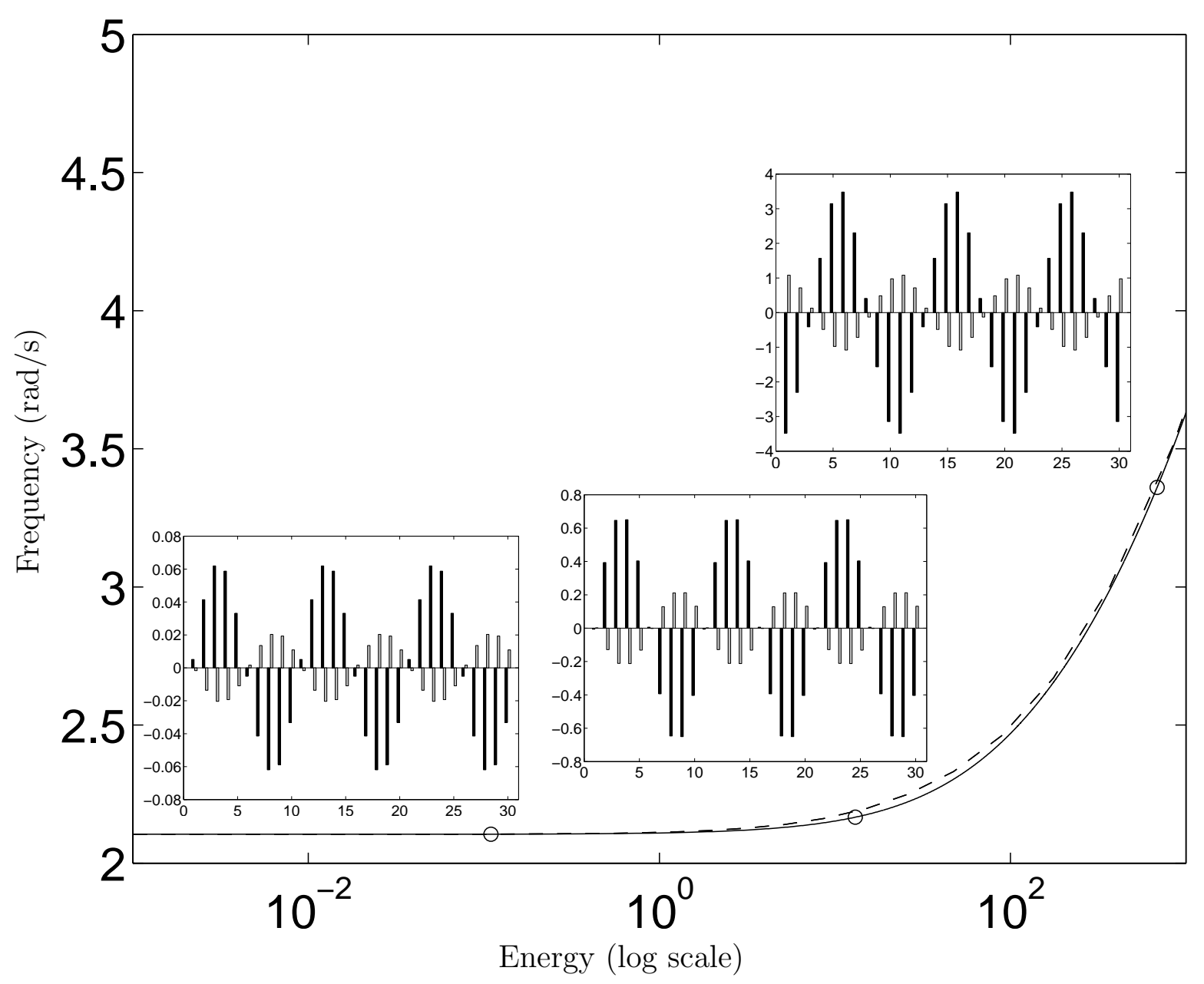

Figure 12: FEP of the traveling-wave NNM corresponding to 1:1 internal resonance between the modes of mode pair $(1,3)$ (solid line). For comparison, the dashed line represents the backbone of one standing-wave NNM of the mode pair $(1,3)$. NNMs represented by bar graphs are inset. 


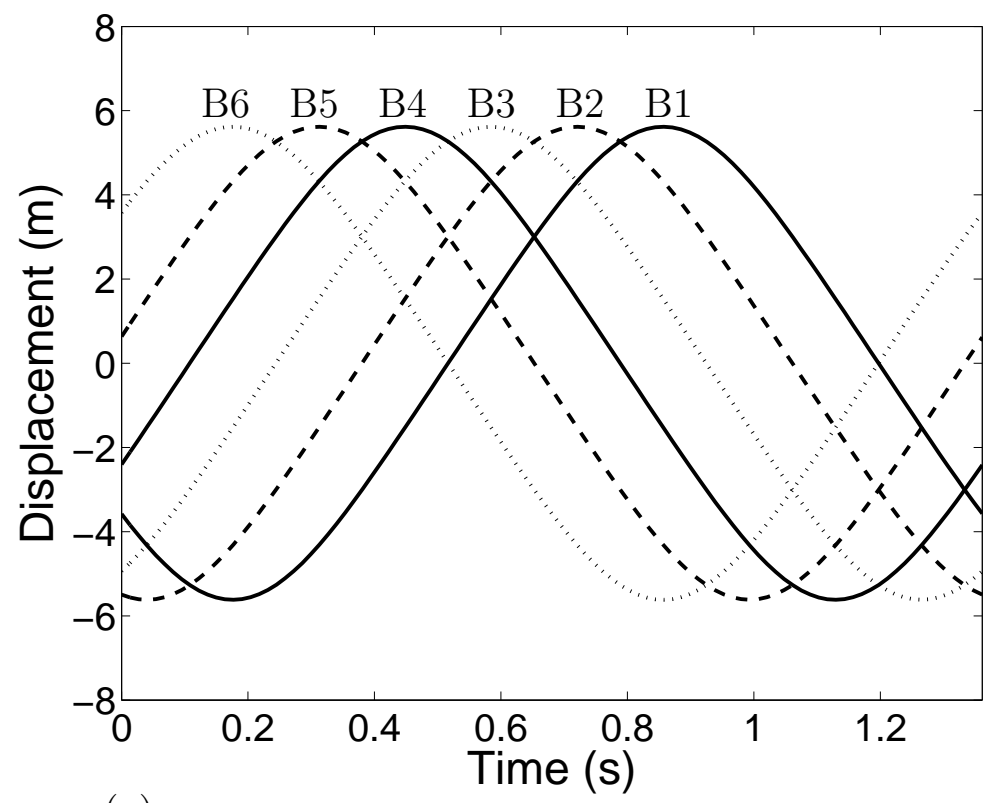

(a)
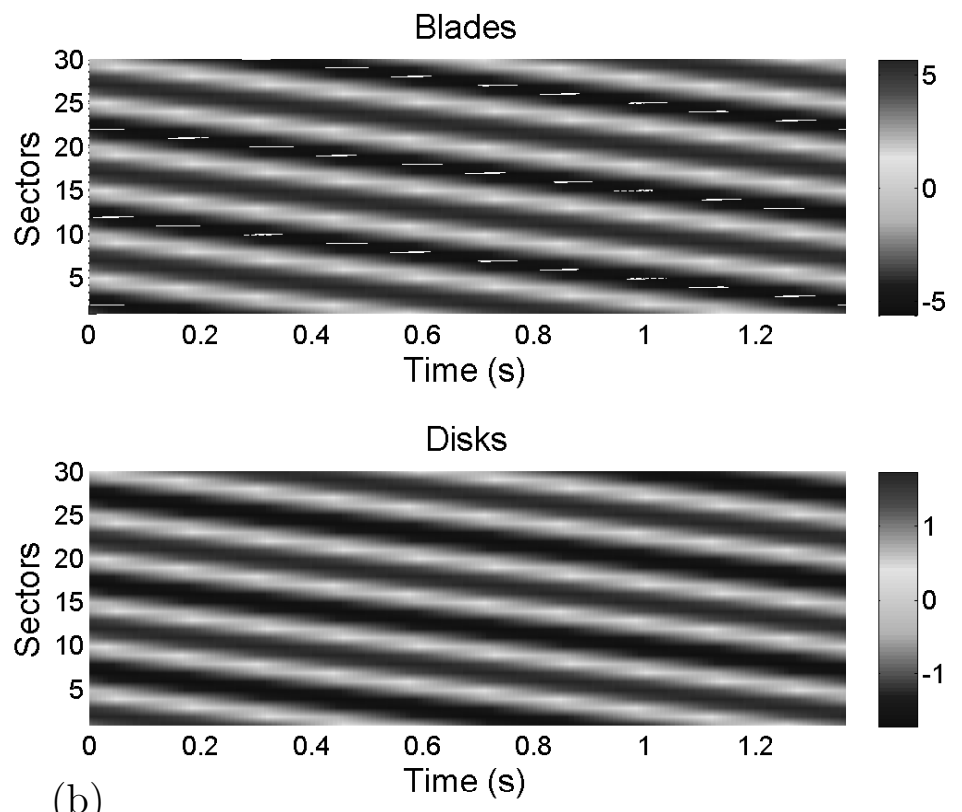

Figure 13: (a) Time series of the first six blades during traveling-wave NNM motion of mode pair (1,3) (see Figure 12); (b) contour plot. 

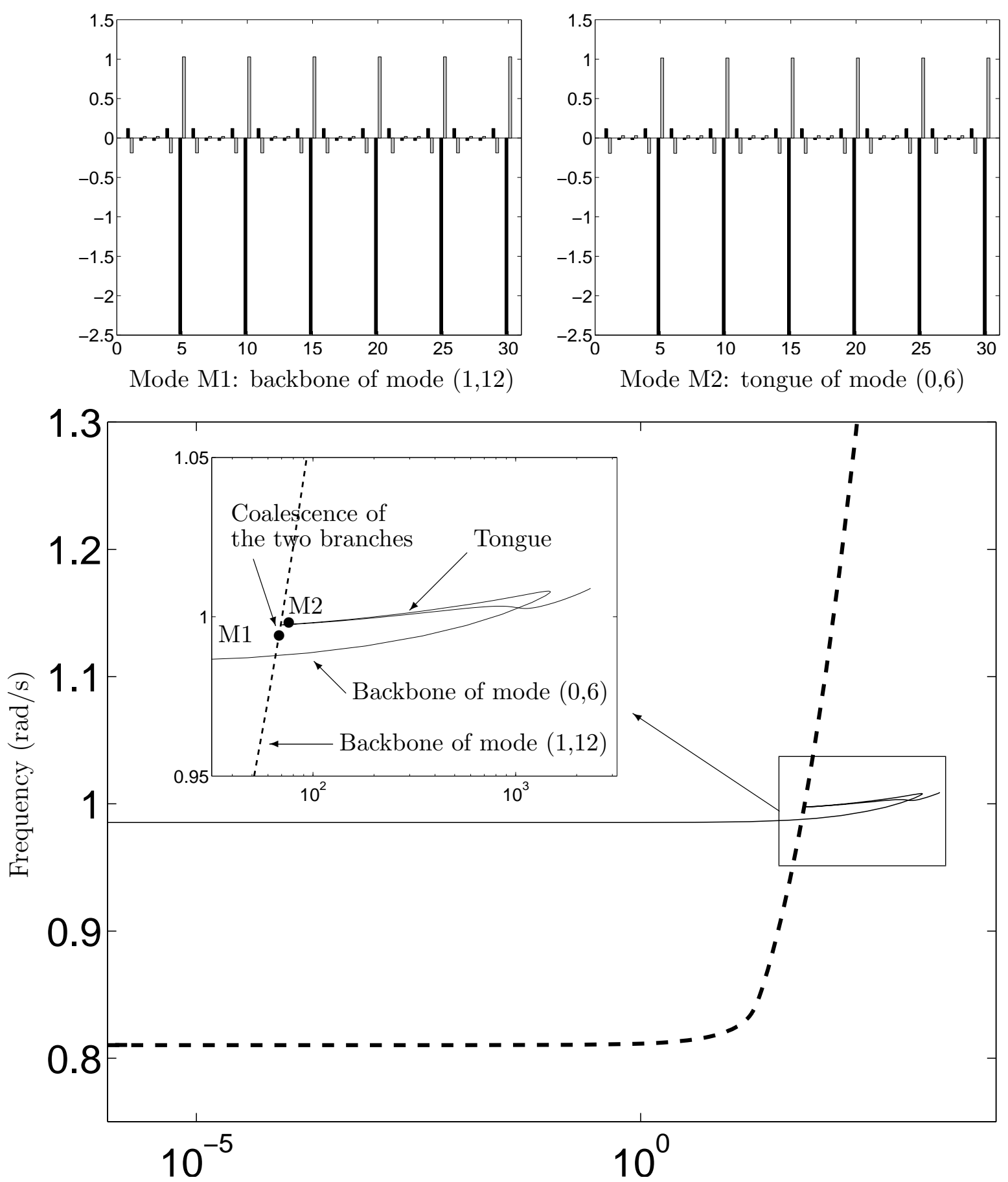

Energy (log scale)

Figure 14: 3:1 internal resonance between modes of the mode pairs $(0,6)$ and $(1,12)$. The solid line corresponds to the backbone of one mode of the mode pair $(0,6)$, which is continued by a tongue of internally resonant NNMs. The dashed line corresponds to the backbone of one mode of the mode pair $(1,12)$ represented at the third of its dominant frequency. 

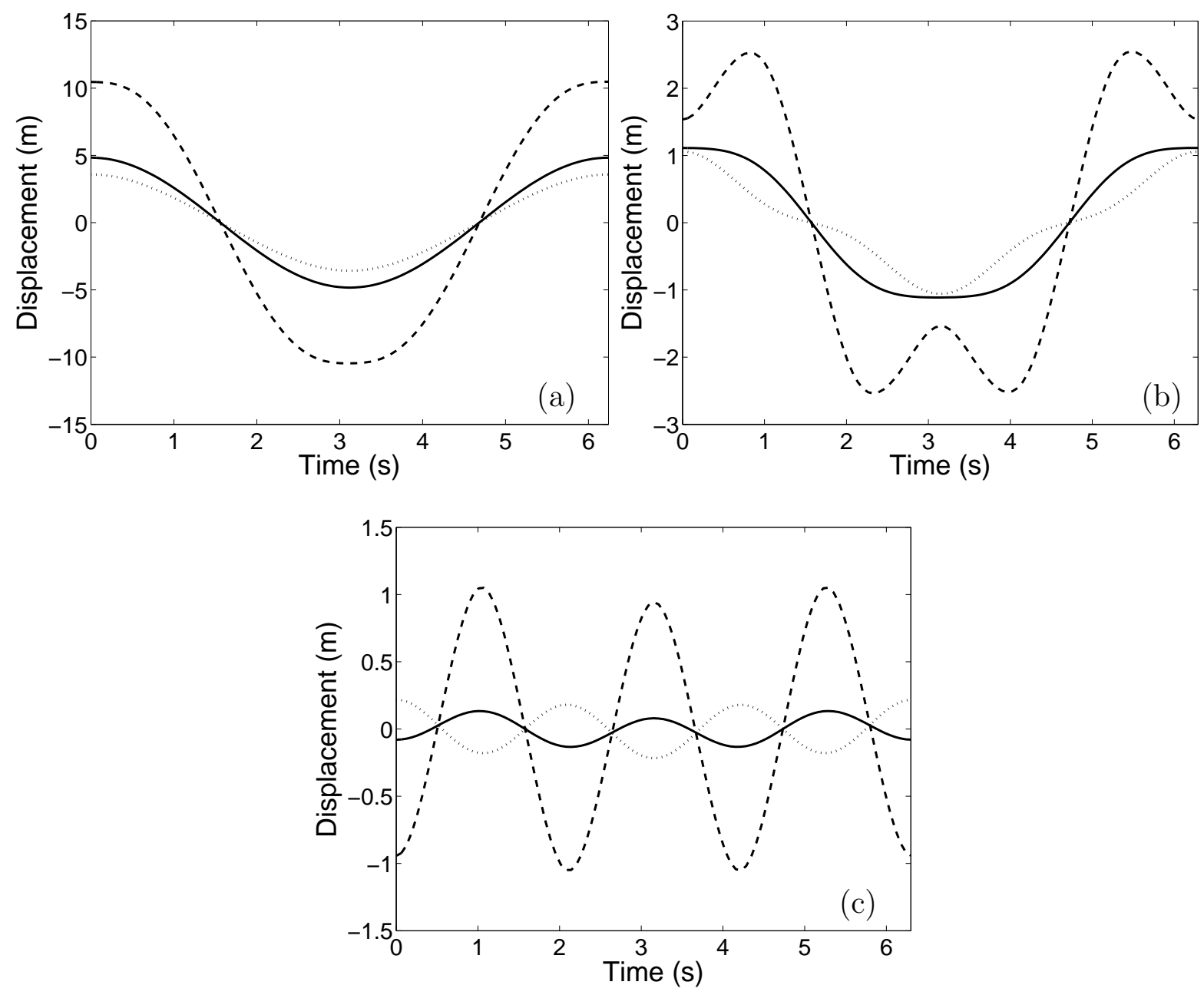

Figure 15: Time series corresponding to NNM motions on the tongue of 3:1 internal resonance (solid line: blade 1; dashed line: disk 10; dotted line: disk 14). (a) Beginning of the tongue (in the vicinity of the branch of mode $(0,6)$ ); (b) middle of the tongue; and (c) extremity of the tongue (in the vicinity of the branch of mode $(1,12)$ ). 


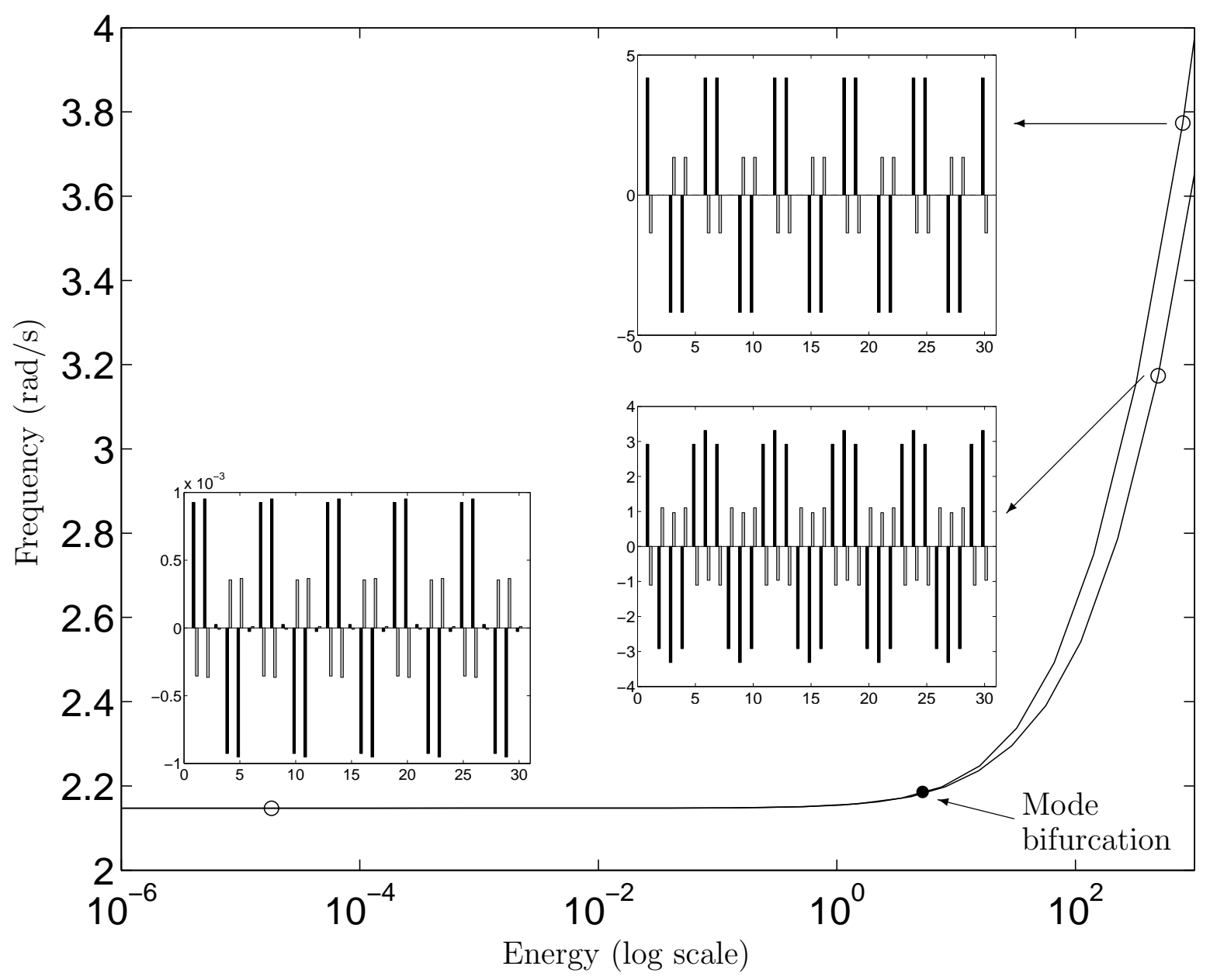

Figure 16: Splitting of one mode of the mode pair $(1,5)$. NNMs represented by bar graphs are inset; they are given in terms of the initial displacements that realize the periodic motion (with zero initial velocities assumed). The blade and disk masses are shown in black and grey, respectively. 

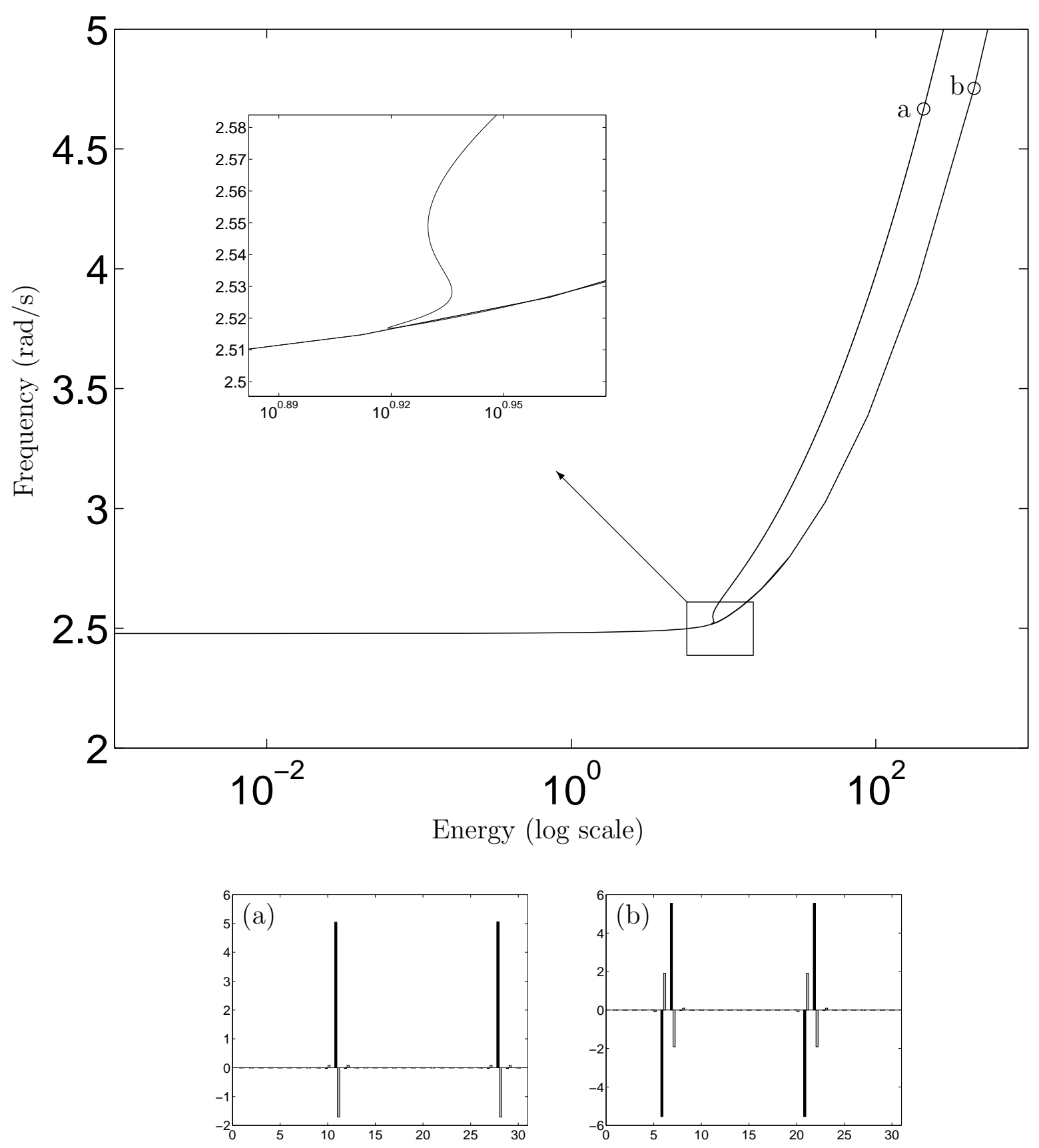

Figure 17: Bifurcation of the mode pair $(1,14)$. 

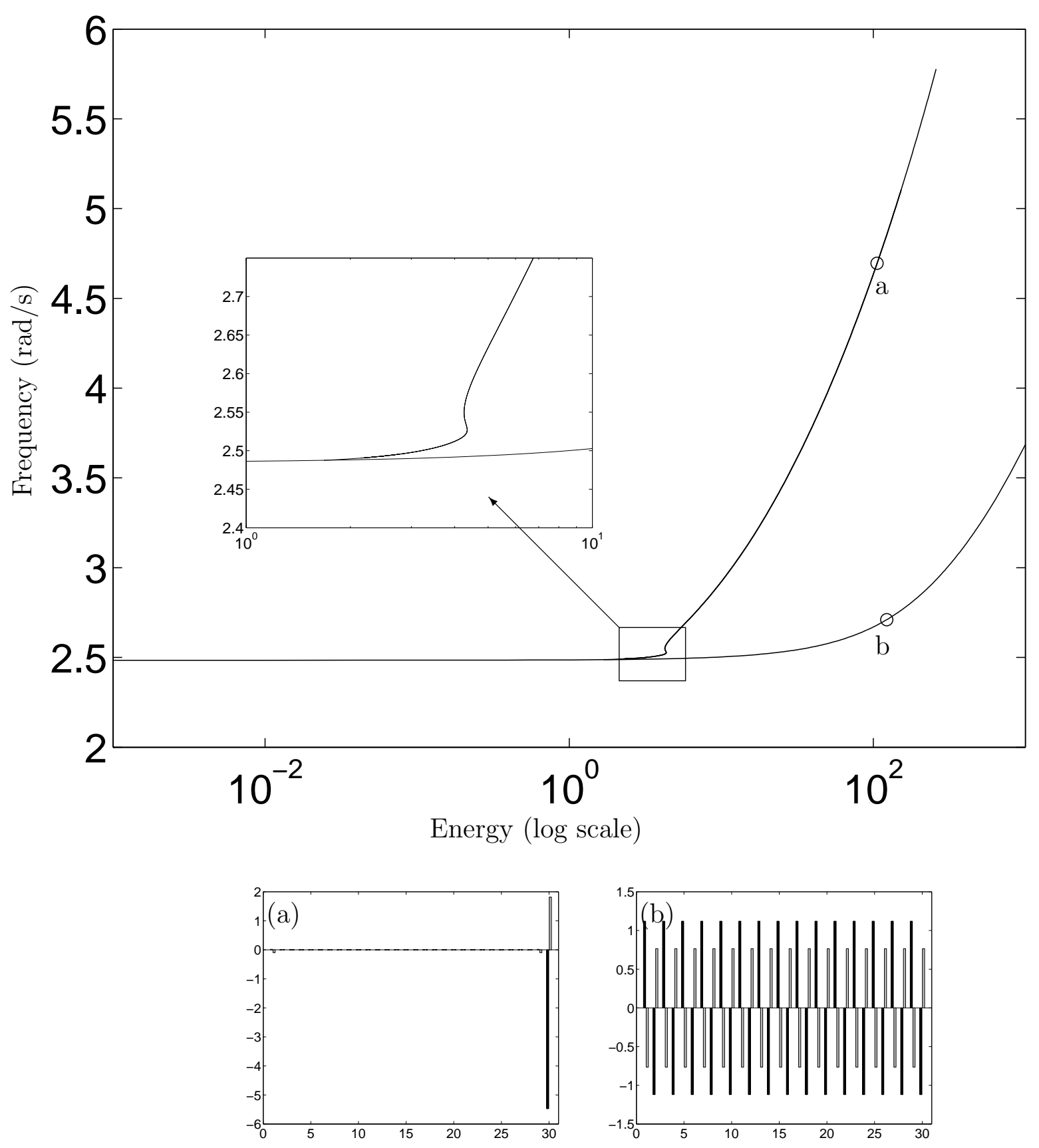

Figure 18: Bifurcation of mode $(1,15)$. 

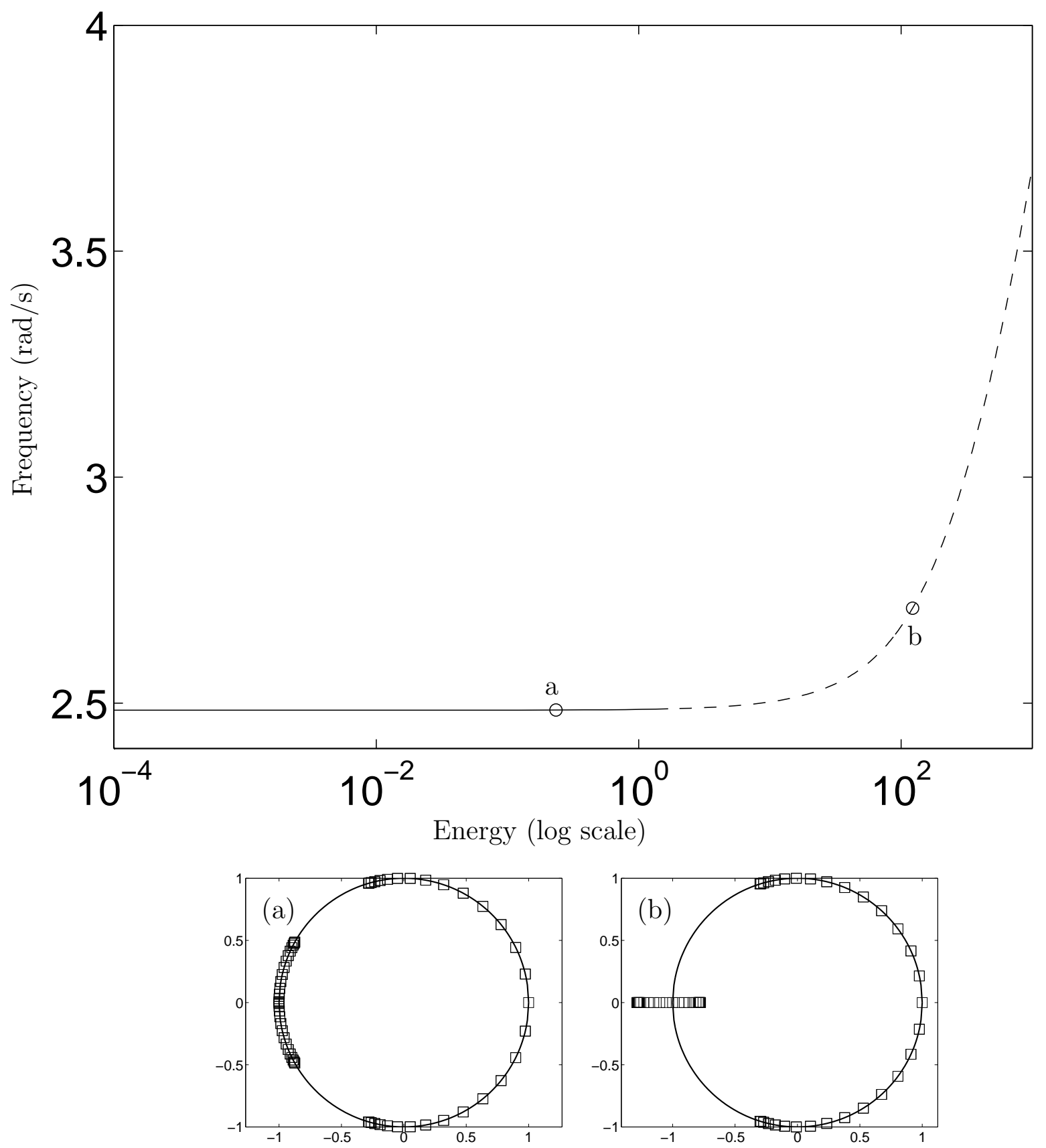

Figure 19: Stability analysis of mode (1,15). Top plot: FEP; a solid (dashed) line indicates stability (instability). Bottom plots: unit circle and Floquet multipliers represented by squares. 